\title{
Extended Super-Kač-Moody Algebras and Their Super-Derivation Algebras
}

\author{
R. Coquereaux, ${ }^{1}$ L. Frappat, ${ }^{2}$ E. Ragoucy ${ }^{2}$ and P. Sorba ${ }^{2}$ \\ ${ }^{1}$ CPT 2, CNRS-Luminy, Case 907, F-13288 Marseille Cedex 09, France \\ ${ }^{2}$ LAPP, B.P. 110, F-74941 Annecy-le-Vieux Cedex, France
}

Received July 6, 1989; in revised form January 20, 1990

\begin{abstract}
We study the $N$-extended super-Kač-Moody algebras, i.e. extensions of the Lie algebra of the loop group over the super-circle $A_{N}$. The extensions are characterized by 2-cocycles which are computed in terms of the cyclic cohomology of the Grassmann algebra with $N$ generators. The graded algebra of super-derivations compatible with each extension is determined. The cases $N=1,2,3$ are examined in detail and their relation with the Ademollo et al. superconformal algebras is discussed. We examine the possibility of defining new superconformal algebras which, for $N>1$, generalize the $N=1$ RamondNeveu-Schwarz algebra.
\end{abstract}

\section{Contents}

1. Introduction . . . . . . . . . . . . . . . . . . . . . 2

2. Central Extensions of the Loop Algebras with $N$ Supersymmetries . . 3 2.1. General Discussion . . . . . . . . . . . . . . . . . 4

2.1.1. Loop Algebras and Super Loop Algebras . . . . . . . 4

2.1.2. Kač-Moody and Super Kač-Moody Algebras . . . . . 4

2.1.3. Cyclic Cohomology of Associative Algebras and Cohomology of Lie Algebras . . . . . . . . . . . . . . . . 6

2.1.4. Hochschild and Cyclic Cohomology of the Algebra of Functions on the Circle . . . . . . . . . . . . . 8

2.1.5. Hochschild and Cyclic Cohomology of Grassmann algebras. 8

2.1.6. Cyclic Cocycles on $G_{N}$. General Results . . . . . . . . . 11

2.1.7. The Cyclic Cohomology of the Super-Circle . . . . . . 13

2.2. Explicit Results. . . . . . . . . . . . . . . . . . . 15

3. Algebras of Super-Derivations (the General Framework). . . . . . 17

3.1. General Discussion . . . . . . . . . . . . . . . . . 17

3.1.1. Derivations and Super-Derivations. . . . . . . . . . 17 
3.1.2. The Compatibility Equation . . . . . . . . . . . . 17

3.1.3. A Problem of Stratification . . . . . . . . . . . . 18

3.2. Study of the Cases $N=1, N=2, N=3$. . . . . . . . . . 18

3.2.1. The Case with One Grassmann Generator . . . . . . . 18

3.2.2. The Case with Two Grassmann Generators . . . . . . 22

3.2.3. The Case with Three Grassmann Generators . . . . . . 23

4. Superconformal Algebras and Algebras of Super-Derivations.

Discussion and Open Problems . . . . . . . . . . . . . . 24

4.1. The Ademollo et al. Algebras. . . . . . . . . . . . . . . . 24

4.1.1. Generalities . . . . . . . . . . . . . . . . . . 24

4.1.2. The Ademollo et al. Algebras as Super-Derivation Algebras 25

4.1.3. The $N=2$ Case . . . . . . . . . . . . . . . . 26

4.2. New Kind of Superconformal Algebras? . . . . . . . . . . . . . 27

4.2.1. Embedding of the $N$-Ademollo et al. Algebra in $\operatorname{Der}\left(A_{N+1}\right) \quad 28$

4.2.2. A Very Particular Chain of Cocycles and Their Corresponding Algebras . . . . . . . . . . . . . . . . . . . 29

5. Conclusion. . . . . . . . . . . . . . . . . . . . . . 30

Appendix . . . . . . . . . . . . . . . . . . . . . . . 30

References . . . . . . . . . . . . . . . . . . . . 35

\section{Introduction}

Supersymmetries in physics have brought the study of graded commutative algebras and graded Lie algebras to the forefront of the arena of theoretical physics in the last 10 years and have also triggered a revival of their interest in pure mathematics. Infinite dimensional Lie algebras and in particular Kač-Moody algebras have also shown their usefulness in quantum field theory, in particular in the realm of two dimensional models and in string theory. The study of supersymmetric string theories leads therefore to the introduction of infinite dimensional superalgebras (from now on, we will use mostly the adjective "super" rather than the traditional " $Z_{2}$-graded" qualifier used by most mathematicians). As it is usually the case in physics, "matter-fields" take their values in a representation space of an algebra (or of a group). What happens here is that most interesting representations happen to be projective representations (they transform correctly only up to a phase); this means that they are genuine representations of a central extension of the algebra. This situation was already recognized in the early days of quantum field theory (presence of Schwinger terms) and has been illustrated many times under different names since. For example, it is well known that central extensions of loop algebras are described by a so-called "central charge," the result being a Kač-Moody algebra, and it is also known that the algebra of derivations of such an algebra can itself be central extended to obtain the so-called Virasoro algebra. When one incorporates supersymmetries, i.e. Grassmann generators into the game, one could expect a similar situation. However, several new phenomena appear.

First, the number of independent "central charges" that one can add is no longer equal to one as was the case previously and this number also depends upon the number of Grassmann generators. In other words, if we start with a given loop algebra and supersymmetrize it by adding $N$ Grassmann generators, it will still 
be possible to construct several inequivalent graded Lie algebras (super-Kač-Moody algebras), each one characterized by the choice of a different central charge. Technically, such a "central charge" is described by the choice of a non-trivial cocycle and the space of these cocycles is a linear space; the inequivalent extensions can therefore be described by choosing a basis in the space of these cocycles. In the first part of this article, we will describe this procedure in detail and relate these cocycles to the cyclic cohomology (see Sect. 2) of the "super-circle" (this denotes the tensor product of the Grassmann algebra with $N$ generators times the (commutative) algebra of functions on the circle). As a by-product - or a necessary tool - we will also describe the Hochschild and cyclic cohomologies of these two algebras. A result of independent interest will be the explicit expression of a basis for the cyclic one-cocycles of a Grassmann algebra using the Berezin integral. We will study explicitly the case $N=1,2$ and 3 and we will also give general formulae for an arbitrary $N$.

Another phenomenon, which is a new feature of the supersymmetric case appears when one studies derivations of functions on the super-circle (the analogue of the algebra of vector fields of the circle). Indeed, an algebra of derivations for a central extension of a loop algebra has to be compatible - of course - with the cocycle defining the extension. This, in turns, is equivalent to selecting a subalgebra of all (super)derivations of superfunctions of the super-circle itself. This procedure has already been followed in [1] where a particular central extension in the case $N=1$ was investigated. Actually, this case corresponds to the usual $N=1$ superconformal algebra. We will devote the second part of the present article to the study of the above mentioned compatibility equations and more generally to the study of (graded) derivations on the central extensions on the super-circle. The particular cases $N=1$ and $N=2$ will be treated explicitly (see Sect. 3).

A class of algebras of particular interest in superstring theories are the so-called "superconformal" (or Ademollo et al.) algebras [2]. In the same way the $N=1$ superconformal (or Ramond-Neveu-Schwarz (RNS)) algebra can be defined as the particular algebra of super-derivations corresponding to a central extension on the $N=1$ super-circle, one may wonder whether the other members of the family of the superconformal algebras of [2] can be characterized in the same way. We will see in Sect. 4 that this is not the case; in particular we will show that there is no non-trivial cocycle compatible with the $N \geqq 2$ superconformal algebra from the $N \geqq 2$ super-circle. We will propose new types of extended algebras, associated with super-Kač-Moody ones and generalizing the $N=1$ RNS algebra.

\section{Central Extensions of the Loop Algebras with $N$ Supersymmetries}

The organization of this section is the following. We start by recalling the definition of loop algebras and super-loop algebras. Then we show in Sect. 2.1.2 how central extensions of these Lie algebras (i.e. Kač-Moody and super-Kač-Moody algebras) are determined by Lie algebra cocycles (or graded Lie Algebra cocycles) of degree two which, in turns, are fully determined by cyclic cocycles of degree one on the algebra $C\left(S^{1}\right)$ of smooth functions on the circle (or on the super-circle). We recast these results in the general framework of "Non-Commutative Geometry": Paragraph 2.1.3 may be skipped in the first reading. In Sect. 2.1.4, we recall the 
description of cyclic cocycles of degree one on $C\left(S^{1}\right)$ and we describe explicitly in Sects. 2.1.5 and 2.1.6 cyclic cocycles of degree one on the Grassmann algebra $G_{N}$ with $N$ generators. Finally, we show how the results of the above sections allow us to compute easily the cyclic cocycles of degree one on the super-circle $A_{N}=C\left(S^{1}\right) \otimes G_{N}($ Sect. 2.1.7).

In Sect. 2.2, we gather explicit results concerning cyclic cocycles for $A_{N}$ in the case $N=1,2,3$.

\subsection{General Discussion}

2.1.1. Loop Algebras and Super Loop Algebras. A loop algebra $L($ Lie $G)$ can be defined as the space of loops in a Lie algebra $\operatorname{Lie}(G)$ with the commutator

$$
[\xi, \eta](p)=[\xi(p), \eta(p)], \quad \xi, \eta \in L(\text { Lie } G), \quad p \in S^{1} .
$$

This Lie algebra can be identified with the Lie algebra Lie $(L G)$ of the loop group $L G$, itself defined $[3,4]$ as the group of smooth maps from $S^{1}$ into a finitedimensional Lie group $G$. As a particular case, we can take $G=U(1)$, then Lie $(G)=R$ and the loop algebra $L(\operatorname{Lie} G)$ can be identified with the space of periodic functions. This algebra is also the commutative algebra of smooth functions on the circle $S^{1}$; it will be denoted ${ }^{1}$ by $C\left(S^{1}\right)$ and will play an important role in the following discussion. Elements of $L(\mathrm{Lie} G)$ can be written as linear combinations of elements of the kind $X^{a}(\bullet) T_{a}$, where $X^{a}(\bullet)$ is a function on the circle, i.e. an element of $C\left(S^{1}\right)$ and $\left\{T_{a}\right\}$ denotes a basis in the Lie algebra $\operatorname{Lie}(G):\left[T_{a}, T_{b}\right]=f_{a b}^{c} T_{c}$.

In this paper, we are mostly interested in the supersymmetric case. In particular, we will replace the circle (or better the commutative algebra $C\left(S^{1}\right)$ ) by the super-circle $A_{N}$ which is the (non-commutative) algebra $C\left(S^{1}\right) \otimes G_{N}$, where $G_{N}$ is the Grassmann algebra with $N$ generators. More generally, to any loop algebra $K$ we can associate a super-loop algebra $K_{N}$ by allowing the functions $X^{a}(\bullet)$ to belong to the super-circle $A_{N}$ rather than to the commutative algebra $C\left(S^{1}\right)$.

2.1.2. Kač-Moody and Super Kač-Moody Algebras. Kač-Moody algebras are central extensions of loop algebras. At the level of Lie algebras, a central extension of such a loop algebra can be written as $\operatorname{Lie}(\hat{L} G)=\operatorname{Lie}(L G) \oplus R$ with the Lie bracket

$$
[(\xi, \alpha),(\eta, \beta)]=([\xi, \eta], \omega(\xi, \eta)) \quad \xi, \eta \in \operatorname{Lie}(L G), \quad \alpha, \beta \in R,
$$

where $\omega(\xi, \eta)$ is a Lie algebra cocycle. Such an object has to be antisymmetric (this comes from the anticommutativity of the Lie bracket) and has to satisfy the following cocycle condition (coming from the fact that the new Lie bracket has to satisfy the Jacobi identity):

$$
\omega([\xi, \eta], \zeta)+\omega([\eta, \zeta], \xi)+\omega([\zeta, \xi], \eta)=0, \quad \xi, \eta, \zeta \in \operatorname{Lie}(L G) .
$$

One then proves [4] that such a cocycle for $G$ compact is necessarily of the kind ${ }^{2}$

$$
\omega(\xi, \eta)=\frac{1}{2 i \pi} \int_{|t|=1} \frac{d t}{t}\left\langle\xi(t), \hat{\partial}_{t} \eta(t)\right\rangle .
$$

\footnotetext{
1 The functions will be taken infinitely differentiable

2 With $t=e^{i \phi}$ all functions are periodic in $\phi$ with period $2 \pi$
} 
From now on, we will use the notation $\hat{\partial}_{t}$ for $t \partial_{t}=t \partial / \partial t$. All the cocycles, and thus all central extensions of $\operatorname{Lie}(L G)$ are therefore completely determined by the choice of a symmetric invariant form $\langle$,$\rangle in \operatorname{Lie}(G)$ (it has to be proportional to the Killing form when $G$ is simple).

This result can be proved in (at least) two ways. The first one [4] consists in making a Fourier expansion of the periodic functions $\xi(t)$ and $\eta(t)$ and in showing that the above expression for $\omega$ is the only possible one compatible with the cocycle condition. Another proof goes along the following lines. One first notices that what really matters is the algebra $C\left(S^{1}\right)$ of smooth functions on the circle $S^{1}$. Indeed the algebra of $n \times n$ matrices with elements in $C\left(S^{1}\right)$ is the tensor product $M_{n}(C) \otimes C\left(S^{1}\right)$ $\left(M_{n}(C)\right.$ denoting the algebra of $n \times n$ matrices on $C$ ). So, a 2 -form $\omega$ on this algebra is fully determined as soon as we know its value on elements of the kind $m \otimes x$ with $m \in M_{n}(C)$ and $x \in C\left(S^{1}\right)$. Let therefore $\omega_{c}$ be an antisymmetric 2-form on the commutative algebra $C\left(S^{1}\right)$, then $\omega=$ Trace $\otimes \omega_{c}$ is an antisymmetric 2-form on the algebra $M_{n}(C) \otimes C\left(S^{1}\right)$. More precisely, $\omega\left(m_{0} \otimes x_{0}, m_{1} \otimes x_{1}\right)=$ Trace $\left(m_{0} m_{1}\right) \omega_{c}\left(x_{0}, x_{1}\right)$. We will explain this construction in more details in Sect. 2.1.3. Moreover, if $\omega_{c}$ satisfy the following condition:

$$
\omega_{c}(x y, z)+\omega_{c}(y z, x)+\omega_{c}(z x, y)=0, \quad x, y, z \in C\left(S^{1}\right),
$$

then, $\omega$ will be a Lie algebra cocycle i.e. will satisfy the condition recalled previously. Such a 2-form $\omega_{c}$ on $C\left(S^{1}\right)$ is called a cyclic cocycle [5] of degree one on the algebra $C\left(S^{1}\right)$ and the above relation between cyclic cocycles and Lie algebra cocycles is a particular case of a general result [6]. We will give more details in what follows on cyclic cohomology but for the moment, it is enough to state the fact that the generator in degree one ${ }^{3}$ of cyclic cohomology for the algebra $C\left(S^{1}\right)$ is precisely given by

$$
\omega_{c}(x, y)=\frac{1}{2 i \pi} \int_{|t|=1} \frac{d t}{t} x(t) \hat{\partial}_{t} y(t) .
$$

It is easy to check that the two properties characterizing a cyclic cocycle are indeed verified (the antisymmetry comes from the integration by part, taking the periodicity into account). The final result about Lie algebra cocycles on the loop algebras then follows.

In order to generalize these ideas to the $Z_{2}$-graded case, we first notice that if $A$ is a graded commutative algebra (such as the Grassmann algebra), one can define the following product in $M_{n}(A) \equiv M_{n}(C) \otimes A$ :

$$
\begin{aligned}
{\left[m_{0} \otimes a_{0}, m_{1} \otimes a_{1}\right] } & \equiv\left[m_{0}, m_{1}\right] \otimes a_{0} a_{1}=m_{0} m_{1} \otimes a_{0} a_{1}-(-1)^{\partial a_{0} \partial a_{1}} m_{1} m_{0} \otimes a_{1} a_{0} \\
& =\left(m_{0} \otimes a_{0}\right)\left(m_{1} \otimes a_{1}\right)-(-1)^{\partial a_{0} \partial a_{1}}\left(m_{1} \otimes a_{1}\right)\left(m_{0} \otimes a_{0}\right),
\end{aligned}
$$

where $\partial a$ denotes the intrinsic $Z_{2}$-grading of the element $a \in A$. It is easy to check that $M_{n}(A)$ satisfies the graded Jacobi identity and is therefore a graded Lie algebra. The rest of the discussion is similar to the previous case: in order to characterize the central extension of super-loop algebras (i.e. super-Kač-Moody algebras), we have to classify the solutions of the equation determining the cocycles. This, in turn, boils down to determine the cyclic cocycles of degree one on the super-circle.

${ }^{3}$ Notice that a cyclic cocycle of degree $p$ is a function of $p+1$ arguments 
In the non-supersymmetric case, there exists only one solution that we mentioned previously. However, in the supersymmetric case, there are many independent solutions (this comes from the fact that the cyclic cohomology of Grassmann algebras is in a sense "richer" than the cyclic cohomology of the algebra $C\left(S^{1}\right)$ ). In the supersymmetric case, the super Lie bracket is no longer anticommutative but still satisfies the equation:

$$
[\xi, \eta]=-(-1)^{\partial \xi \partial \eta}[\eta, \xi] .
$$

Also the super-Jacobi identity reads:

$$
(-1)^{\partial \xi \partial \eta}[\xi,[\zeta, \eta]]+(-1)^{\partial \xi \xi \zeta}[\zeta,[\eta, \xi]]+(-1)^{\partial \eta \partial \zeta}[\eta,[\xi, \zeta]]=0 .
$$

Consequently, the cocycle $\omega$ determining the extension is no longer antisymmetric but graded antisymmetric and has to satisfy a $Z_{2}$-graded version of the cocycle condition. These two equations read:

$$
\omega(\xi, \eta)=-(-1)^{\partial \xi \partial \eta} \omega(\eta, \xi)
$$

and

$$
(-1)^{\partial \xi \partial \eta} \omega(\xi,[\zeta, \eta])+(-1)^{\partial \zeta \partial \xi} \omega(\zeta,[\eta, \xi])+(-1)^{\partial \eta \partial \zeta} \omega(\eta,[\xi, \zeta])=0 .
$$

In order to discuss these equations, one has to use the relation between the cohomology of (super)Lie algebras and the $\left(Z_{2}\right.$-graded) cyclic cohomology of the non-commutative (but associative) algebra $A_{N}=C\left(S^{1}\right) \otimes G_{N}$, i.e. the super-circle. A cyclic cocycle of degree one $\Phi$ on the super-circle has to satisfy the following conditions:

$$
\begin{gathered}
\Phi(Y, X)=-(-1)^{\partial X \partial Y} \Phi(X, Y), \\
-\Phi(X, Y Z)+\Phi(X Y, Z)+(-1)^{\partial Z(\partial X+\partial Y)} \Phi(Z X, Y)=0,
\end{gathered}
$$

where $X, Y, Z \in A_{N}$. We will discuss the solution of these equations in Sect. 2.1.7.

2.1.3. Cyclic Cohomology of Associative Algebras and Cohomology of Lie Algebras. We already gave the definition of cyclic cocycles of degree one in the previous paragraph and we will not need more for our purpose. However, it may be interesting to recast our present problem in the more general framework of "Non-Commutative Geometry." We will first define Hochschild cohomology and cyclic cohomology for associative algebras and then make the link with the cohomology of algebras of matrices.

Let $A$ be an associative algebra (not necessarily commutative) which we will take $Z_{2}$ graded. Then, complex (or real) valued $n$-linear forms on $A$ are particularly interesting objects to consider. A Hochschild cochain of degree $n$ is defined as a multilinear form $\Phi\left(a_{0}, a_{1}, \ldots, a_{n}\right)$ of degree $n+1 .{ }^{4}$ We define the following operator $b$ from cochains of degree $n$ to cochains of degree $n+1$ :

$$
\begin{aligned}
{[b \Phi]\left(a_{0}, \ldots, a_{n}, a_{n+1}\right)=} & \sum_{j=0}^{n}(-1)^{j} \Phi\left(a_{0}, \ldots, a_{j} a_{j+1}, \ldots, a_{n+1}\right) \\
& +(-1)^{n+1}(-1)^{\varepsilon_{n+1}} \Phi\left(a_{n+1} a_{0}, a_{1}, \ldots, a_{n}\right),
\end{aligned}
$$

${ }^{4}$ Actually, there are several kinds of Hochschild complexes depending upon the choice of a module for $A$. Here, the module is the dual of the algebra itself 
where $\varepsilon_{n+1}=\partial a_{n+1} \sum_{i=0}^{n} \partial a_{i}$. It is not too difficult to show that $b^{2}=0$. This operator $b$ is called the $Z_{2}$-graded Hochschild coboundary operator and the corresponding cohomology, $Z_{2}$-graded Hochschild cohomology: Hochschild cocycles $\Phi$ are such that $b \Phi=0$, Hochschild coboundaries $\Psi$ are such that $\Psi=b \Phi$ for some $\Phi$. The space of Hochschild cocycles of degree $n$ modulo Hochschild coboundaries is the Hochschild cohomology group of degree $n$.

The cyclicity operator $\lambda$ is defined as follows: ${ }^{5}$

$$
[\lambda \Phi]\left(a_{0}, a_{1}, \ldots, a_{n}\right)=(-1)^{n}(-1)^{\varepsilon_{n}} \Phi\left(a_{n}, a_{0}, \ldots, a_{n-1}\right) .
$$

The multilinear form $\Phi$ is called cyclic whenever $\lambda \Phi=\Phi$. It can be shown [5] that if $\Phi$ is cyclic, so is $b \Phi$. Therefore it is tempting to consider the subcomplex of Hochschild cochains built out of cyclic cochains. One defines therefore the space of cyclic cocycles (i.e. Hochschild cocycles which are cyclic) and the space of cyclic coboundaries (they are of the kind $b \Phi$ for some cyclic cochain $\Phi$ ). The quotient of cyclic cocycles of degree $n$ modulo cyclic coboundaries defines the cyclic cohomology group of degree $n$. The theory of cyclic cohomology has been introduced and developed in [5]; the $Z_{2}$-graded case has been particularly studied in [7]; for a general review, see [8].

Given an algebra $A$, it becomes possible to consider matrices with elements in $A$ (such an algebra $M_{n}(A)$ is obtained by taking the tensor product of $A$ with the algebra of $n \times n$ matrices over the complex numbers). As we saw in Sect. 2.1.2, in the case where $A$ is (graded)commutative, it is possible to define a (graded)Lie bracket on $M_{n}(A)$. Cohomology for Lie algebras of matrices is well known and we will not recall the definitions here (see for instance [9]). The main idea is that it is possible to relate the cohomology of Lie algebras to the cyclic cohomology of $A$ itself. Notice first that, by linearity, a Lie algebra cocycle will be defined as soon as we know its definition on elementary matrices (which are of the kind $M_{j}^{i} \otimes a$, where $a \in A$ and where the only non-zero entry of $M_{j}^{i}$ is 1 in position $\left.(i, j)\right)$. The result (due to [6]) is the following. Let $\Phi$ be a cyclic cocycle on $A$, that we suppose commutative; in order to get a multilinear form on $M_{n}(C) \otimes A$, one composes it with the trace operator. The object that we obtain is then "cyclically antisymmetric" but not fully antisymmetric. Therefore, in order to get a multilinear antisymmetric form on the Lie algebra, we have to fully antisymmetrize it:

$$
\omega\left(m_{0} \otimes a_{0}, \ldots, m_{n} \otimes a_{n}\right)=\operatorname{Ant}\left(\operatorname{Trace}\left(m_{0} \cdots m_{n}\right) \Phi\left(a_{0}, \ldots, a_{n}\right)\right) .
$$

The result $\omega$ is a Lie algebra cocycle. ${ }^{6}$

In the case where we start from a graded commutative algebra rather than a commutative one, and from a super Lie algebra rather than a Lie algebra, the results are analogous.

In the present situation, we are only interested in Lie algebra cocycles of degree two - since they determine central extensions - for a Lie algebra of matrices whose elements are functions on the super-circle. We have therefore to determine cyclic

\footnotetext{
5 The notation $\lambda$ differs by a sign from the one introduced in [5]

${ }^{6}$ More precisely, cyclic cohomology coincides with the (primitive part of the) cohomology of the Lie algebra of matrices
} 
cocycles of degree one on the algebra $A_{N}=C\left(S^{1}\right) \otimes G_{N}$. Notice that for cyclic cocycles of degree one, we do not have to antisymmetrize, since, at this order, cyclic antisymmetry coincides with full antisymmetry.

2.1.4. Hochschild and Cyclic Cohomology of the Algebra of Functions on the circle. As already stated in Sect 2.1.1, it is rather straightforward to check the following results.

1. The cyclic cohomology group of degree zero coincides with the Hochschild cohomology group in the same dimension and with the space of distributions on the circle $S^{1}$.

2. Cyclic and Hochschild cohomology groups also coincide in degree one (cf. Eqs. (11) and (12)) and all the cocycles are of the kind $a \Phi$, where $a$ is an arbitrary constant and $\Phi=\omega_{c}(x, y)$ is given by formula $(6){ }^{7}$

2.1.5. Hochschild and 'Cyclic Cohomology of Grassmann Algebras $G_{N}$ with $N=1,2,3$. Before studying the cocycles on the super-circle, it is necessary to study those on Grassmann algebras. To our knowledge, these cyclic cocycles have not been explicitly computed in the mathematical literature, only [10] determines the dimension of the cohomology groups of degree $k$ (but ref. [10] does not give their explicit expression). We will therefore discuss quite explicitly the cases $N=1,2$ and 3 before considering the general case.

- Cyclic cocycles of degree 0 and 1 on $G_{1}$

$G_{1}$ is generated by 1 and $\theta$ with $\theta^{2}=0$. According to the previous discussion, cyclic cocycles of degree zero are generated by the two elements $\vartheta_{1}$ and $\vartheta_{\theta}$ with

$$
\vartheta_{1}(1)=1, \quad \vartheta_{1}(\theta)=0, \quad \vartheta_{\theta}(1)=0, \quad \vartheta_{\theta}(\theta)=1 .
$$

Cyclic cocycles of degree one on $G_{1}$ have to satisfy the two following conditions (already given previously):

$$
\varphi(Y, X)=(-1)^{\partial X \partial Y} \varphi(X, Y)
$$

and

$$
-\varphi(X, Y Z)+\varphi(X Y, Z)+(-1)^{\partial Z(\partial X+\partial Y)} \varphi(Z X, Y)=0,
$$

where $X, Y, Z \in G_{1}$. The last equation gives in fact $2^{3}$ equations since $X, Y, Z$ have to be chosen in the set $\{1, \theta\}$. The solution of this system leads to the following result. There is only one cyclic cocycle of degree one in $G_{1}$. We will denote it by $\varphi$. It is characterized by the equations

$$
\varphi(\theta, \theta)=1, \quad \varphi(\theta, 1)=\varphi(1, \theta)=\varphi(1,1)=0 .
$$

It is possible to write the previous cocycle by using a Berezin integral. Let $X, Y \in G_{1}$,

\footnotetext{
7 The general result for the algebra $C(\mathscr{M})$ of smooth functions on a smooth manifold $\mathscr{M}$ is the following [5]: The Hochschild cohomology group of degree $k$ is canonically isomorphic with the space of de Rham currents (i.e. distributional forms) of dimension $k$ on $\mathscr{M}$ and the cyclic cohomology group of degree $k$ is cannonically isomorphic to the direct sum $\operatorname{Ker} \partial \oplus H_{k-2} \oplus$ $H_{k-4} \oplus \cdots$, where $\operatorname{Ker} \partial$ is the space of closed $k$-dimensional currents and where $H_{k}$ denotes the usual de Rham homology of $\mathscr{M}$
} 
then $\varphi(X, Y)=\int X \frac{d}{d \theta} Y d \theta$. Indeed, if we set $X=X_{0} 1+X_{1} \theta$ and $Y=Y_{0} 1+Y_{1} \theta$, then $\varphi(X, Y)=X_{1} Y_{1}$.

- Cyclic cocycles of degree 0 and 1 on $G_{2}$

$G_{2}$ is generated by $1, \theta^{1}$ and $\theta^{2}$ with $\left(\theta^{1}\right)^{2}=0,\left(\theta^{2}\right)^{2}=0$ and $\theta^{1} \theta^{2}=-\theta^{2} \theta^{1}$. According to the previous discussion, cyclic cocycles of degree zero are generated by the four elements $\vartheta_{1}, \vartheta_{\theta^{1}}, \vartheta_{\theta^{2}}, \vartheta_{\theta^{1} \theta^{2}}$. These one-forms are dual to the basis $\left\{1, \theta^{1}, \theta^{2}, \theta^{1} \theta^{2}\right\}$. Notice that $\vartheta_{1}, \vartheta_{\theta^{1} \theta^{2}}$ are even cocycles whereas $\vartheta_{\theta^{1}}, \vartheta_{\theta^{2}}$ are odd.

Cyclic cocycles of degree one are still determined by the two equations given previously; however, the second equation gives in fact $4^{3}=64$ equations since $X, Y, Z$ have to be chosen in the set $\left\{1, \theta^{1}, \theta^{2}, \theta^{1} \theta^{2}\right\}$. This time, one finds five independent cyclic cocycles (which can be taken as generators). The generators will be denoted by $\varphi_{1}, \varphi_{2}, \varphi_{3}, \varphi_{4}, \varphi_{5}$ and are explicitly given as follows (the first three are even and the last two are odd): $\varphi_{1}\left(\theta^{1}, \theta^{1}\right)=1, \varphi_{2}\left(\theta^{2}, \theta^{2}\right)=1, \varphi_{3}\left(\theta^{1}, \theta^{2}\right)=$ $\varphi_{3}\left(\theta^{2}, \theta^{1}\right)=1, \varphi_{4}\left(\theta^{1}, \theta^{1} \theta^{2}\right)=-\varphi_{4}\left(\theta^{1} \theta^{2}, \theta^{1}\right)=1, \varphi_{5}\left(\theta^{2}, \theta^{1} \theta^{2}\right)=-\varphi_{5}\left(\theta^{1} \theta^{2}, \theta^{2}\right)=1$. In the above, we only gave those non-trivial relations which are not a direct consequence of linearity or of elementary rules of calculation in $G_{2}$, like for instance $\varphi_{4}\left(\theta^{1}, \theta^{1} \theta^{2}\right)=-\varphi_{4}\left(\theta^{1}, \theta^{2} \theta^{1}\right)$. All other $\varphi_{i}(X, Y)$ are zero (for instance $\varphi_{2}\left(\theta^{1}, \theta^{2}\right)=0$ ).

It is possible to write the previous cocycles of degree one by using a Berezin integral. Let $X, Y \in G_{2}$, and write $X=X_{0}+X_{1} \theta^{1}+X_{2} \theta^{2}+X_{12} \theta^{1} \theta^{2}$ (same thing for $Y$ ). One gets (using the obvious notation $\partial_{i}=\partial / \partial \theta^{i}$ ):

$$
\begin{aligned}
& \varphi_{1}(X, Y)=X_{1} Y_{1}=\int X\left[\theta^{2} \partial_{1}\right] Y d \theta^{1} d \theta^{2} \\
& \varphi_{2}(X, Y)=X_{2} Y_{2}=-\int X\left[\theta^{1} \partial_{2}\right] Y d \theta^{1} d \theta^{2} \\
& \varphi_{3}(X, Y)=X_{1} Y_{2}+X_{2} Y_{1}=\int X\left[-\theta^{1} \partial_{1}+\theta^{2} \partial_{2}\right] Y d \theta^{1} d \theta^{2} \\
& \varphi_{4}(X, Y)=X_{1} Y_{12}-X_{12} Y_{1}=\int X\left[-\partial_{1}+2 \theta^{2} \partial_{2} \partial_{1}\right] Y d \theta^{1} d \theta^{2} \\
& \varphi_{5}(X, Y)=X_{2} Y_{12}-X_{12} Y_{2}=\int X\left[-\partial_{2}-2 \theta^{2} \partial_{2} \partial_{1}\right] Y d \theta^{1} d \theta^{2}
\end{aligned}
$$

The reader can easily check that the previous one-cocycles are independent and non-trivial, i.e. they are not cyclic coboundaries, by computing explicitly the coboundaries $b \vartheta_{I}$ with $I \in\left\{1, \theta^{1}, \theta^{2}, \theta^{1} \theta^{2}\right\}$, i.e. writing $\left[b \vartheta_{I}\right](X, Y)=\vartheta_{I}(X Y)$. Notice that the last two cocycles involve differential operators of degree two in the Grassmann algebra; however, by introducing the grading of $X$ and $Y$, we will see that they can be written in terms of differential operators of degree one only. The previous cocycles can be written as follows:

$$
\begin{aligned}
& \varphi_{1}(X, Y)=(-1)^{\partial X \partial Y} \int\left[\theta^{2} \partial_{1} Y\right] X d \theta^{1} d \theta^{2}, \\
& \varphi_{2}(X, Y)=-(-1)^{\partial X \partial Y} \int\left[\theta^{1} \partial_{2} Y\right] X d \theta^{1} d \theta^{2}, \\
& \varphi_{3}(X, Y)=(-1)^{\partial X \partial Y} \int\left[\left(-\theta^{1} \partial_{1}+\theta^{2} \partial_{2}\right) Y\right] X d \theta^{1} d \theta^{2}, \\
& \varphi_{4}(X, Y)=-(-1)^{\partial X \partial Y} \int\left[\partial_{1} Y\right] X d \theta^{1} d \theta^{2}, \\
& \varphi_{5}(X, Y)=-(-1)^{\partial X \partial Y} \int\left[\partial_{2} Y\right] X d \theta^{1} d \theta^{2}
\end{aligned}
$$

One can remark that the $Z_{2}$-grading of $\varphi$ is given by the $Z_{2}$-grading of the differential operator which defines it.

- Cyclic cocycles of degree 0 and 1 on $G_{3}$ 
For $G_{3}$, we can choose the basis $\left\{1, \theta^{1}, \theta^{2}, \theta^{3}, \theta^{1} \theta^{2}, \theta^{2} \theta^{3}, \theta^{3} \theta^{1}, \theta^{1} \theta^{2} \theta^{3}\right\}$. Again, cyclic cocycles of degree zero are generated by the dual basis (this space has now dimension $2^{3}=8$ ). These elements will be denoted as follows:

$$
\vartheta_{1}, \vartheta_{\theta^{1}}, \vartheta_{\theta^{2}}, \vartheta_{\theta^{3}}, \vartheta_{\theta^{1} \theta^{2}}, \vartheta_{\theta^{2} \theta^{3}}, \vartheta_{\theta^{3} \theta^{1}}, \vartheta_{\theta^{1} \theta^{2} \theta^{3}}
$$

Cyclic cocycles of degree one have to satisfy the cyclicity condition $\left(8^{2}=64\right.$ equations) and the Hochschild condition $\left(8^{3}=512\right.$ equations). One finds that this vector space has dimension 17 and is generated by the following cocycles (the first nine - that we will denote by $\varphi_{i}$ - are even and the last eight - that we will denote by $\psi_{i}-$ are odd). They are explicitly given as follows (i.e. they are non-vanishing only on the following arguments):

$$
\begin{aligned}
\varphi_{1}\left(\theta^{1}, \theta^{1}\right) & =\varphi_{2}\left(\theta^{2}, \theta^{2}\right)=\varphi_{3}\left(\theta^{3}, \theta^{3}\right)=1, \\
\varphi_{4}\left(\theta^{1}, \theta^{2}\right) & =\varphi_{4}\left(\theta^{2}, \theta^{1}\right)=\varphi_{5}\left(\theta^{2}, \theta^{3}\right)=\varphi_{5}\left(\theta^{3}, \theta^{2}\right)=\varphi_{6}\left(\theta^{3}, \theta^{1}\right)=\varphi_{6}\left(\theta^{1}, \theta^{3}\right)=1, \\
\varphi_{7}\left(\theta^{1}, \theta^{1} \theta^{2} \theta^{3}\right) & =\varphi_{7}\left(\theta^{1} \theta^{2} \theta^{3}, \theta^{1}\right)=\varphi_{7}\left(\theta^{1} \theta^{2}, \theta^{3} \theta^{1}\right)=-\varphi_{7}\left(\theta^{3} \theta^{1}, \theta^{1} \theta^{2}\right)=1, \\
\varphi_{8}\left(\theta^{2}, \theta^{1} \theta^{2} \theta^{3}\right) & =\varphi_{8}\left(\theta^{1} \theta^{2} \theta^{3}, \theta^{2}\right)=-\varphi_{8}\left(\theta^{1} \theta^{2}, \theta^{2} \theta^{3}\right)=\varphi_{8}\left(\theta^{2} \theta^{3}, \theta^{1} \theta^{2}\right)=1, \\
\varphi_{9}\left(\theta^{3}, \theta^{1} \theta^{2} \theta^{3}\right) & =\varphi_{9}\left(\theta^{1} \theta^{2} \theta^{3}, \theta^{3}\right)=-\varphi_{9}\left(\theta^{3} \theta^{1}, \theta^{2} \theta^{3}\right)=\varphi_{9}\left(\theta^{2} \theta^{3}, \theta^{3} \theta^{1}\right)=1, \\
\psi_{1}\left(\theta^{1}, \theta^{1} \theta^{2}\right) & =-\psi_{1}\left(\theta^{1} \theta^{2}, \theta^{1}\right)=1, \\
\psi_{2}\left(\theta^{1}, \theta^{3} \theta^{1}\right) & =-\psi_{2}\left(\theta^{3} \theta^{1}, \theta^{1}\right)=1, \\
\psi_{3}\left(\theta^{2}, \theta^{2} \theta^{3}\right) & =-\psi_{3}\left(\theta^{2} \theta^{3}, \theta^{2}\right)=1, \\
\psi_{4}\left(\theta^{2}, \theta^{1} \theta^{2}\right) & =-\psi_{4}\left(\theta^{1} \theta^{2}, \theta^{2}\right)=1, \\
\psi_{5}\left(\theta^{3}, \theta^{3} \theta^{1}\right) & =-\psi_{5}\left(\theta^{3} \theta^{1}, \theta^{3}\right)=1, \\
\psi_{6}\left(\theta^{3}, \theta^{2} \theta^{3}\right) & =-\psi_{6}\left(\theta^{2} \theta^{3}, \theta^{3}\right)=1 .
\end{aligned}
$$

The last two, $\psi_{7}$ and $\psi_{8}$ have to be independent and should vanish as follows

$$
-\psi\left(\theta^{1}, \theta^{2} \theta^{3}\right)+\psi\left(\theta^{1} \theta^{2}, \theta^{3}\right)+\psi\left(\theta^{3} \theta^{1}, \theta^{2}\right)=0 .
$$

As in the previous case, it is easy to rewrite the cocycles of degree one by using a Berezin integral. Let $X, Y \in G_{3}$ and write the following decomposition on the Grassmann variables $X=X_{0}+X_{1} \theta^{1}+X_{2} \theta^{2}+X_{3} \theta^{3}+X_{12} \theta^{1} \theta^{2}+X_{23} \theta^{2} \theta^{3}+$ $X_{31} \theta^{3} \theta^{1}+X_{123} \theta^{1} \theta^{2} \theta^{3}$ (and the same thing for $Y$ ). One obtains therefore

$$
\begin{aligned}
& \varphi_{1}(X, Y)=X_{1} Y_{1}=-(-1)^{\partial X \partial Y} \int\left[\theta^{2} \theta^{3} \partial_{1} Y\right] X d \theta^{1} d \theta^{2} d \theta^{3}, \\
& \varphi_{2}(X, Y)=X_{2} Y_{2}=-(-1)^{\partial X \partial Y} \int\left[\theta^{3} \theta^{1} \partial_{2} Y\right] X d \theta^{1} d \theta^{2} d \theta^{3}, \\
& \varphi_{3}(X, Y)=X_{3} Y_{3}=-(-1)^{\partial X \partial Y} \int\left[\theta^{1} \theta^{2} \partial_{3} Y\right] X d \theta^{1} d \theta^{2} d \theta^{3}, \\
& \varphi_{4}(X, Y)=X_{1} Y_{2}+X_{2} Y_{1}=-(-1)^{\partial X \partial Y} \int\left[\left(\theta^{3} \theta^{1} \partial_{1}-\theta^{3} \theta^{2} \partial_{2}\right) Y\right] X d \theta^{1} d \theta^{2} d \theta^{3}, \\
& \varphi_{5}(X, Y)=X_{2} Y_{3}+X_{3} Y_{2}=-(-1)^{\partial X \partial Y} \int\left[\left(\theta^{1} \theta^{2} \partial_{2}-\theta^{1} \theta^{3} \partial_{3}\right) Y\right] X d \theta^{1} d \theta^{2} d \theta^{3}, \\
& \varphi_{6}(X, Y)=X_{3} Y_{1}+X_{1} Y_{3}=-(-1)^{\partial X \partial Y} \int\left[\left(\theta^{2} \theta^{3} \partial_{3}-\theta^{2} \theta^{1} \partial_{1}\right) Y\right] X d \theta^{1} d \theta^{2} d \theta^{3}, \\
& \varphi_{7}(X, Y)=X_{1} Y_{123}+X_{123} Y_{1}+X_{12} Y_{31}-X_{31} Y_{12}=-(-1)^{\partial X \partial Y} \int\left[\partial_{1} Y\right] X d \theta^{1} d \theta^{2} d \theta^{3}, \\
& \varphi_{8}(X, Y)=X_{2} Y_{123}+X_{123} Y_{2}+X_{23} Y_{12}-X_{12} Y_{23}=-(-1)^{\partial X \partial Y} \int\left[\partial_{2} Y\right] X d \theta^{1} d \theta^{2} d \theta^{3}, \\
& \varphi_{9}(X, Y)=X_{3} Y_{123}+X_{123} Y_{3}+X_{23} Y_{31}-X_{31} Y_{23}=-(-1)^{\partial X \partial Y} \int\left[\partial_{3} Y\right] X d \theta^{1} d \theta^{2} d \theta^{3}, \\
& \psi_{1}(X, Y)=X_{1} Y_{12}-X_{12} Y_{1}=-(-1)^{\partial X \partial Y} \int\left[\theta^{3} \partial_{1} Y\right] X d \theta^{1} d \theta^{2} d \theta^{3}, \\
& \psi_{2}(X, Y)=X_{1} Y_{31}-X_{31} Y_{1}=-(-1)^{\partial X \partial Y} \int\left[\theta^{2} \partial_{1} Y\right] X d \theta^{1} d \theta^{2} d \theta^{3}, \\
& \psi_{3}(X, Y)=X_{2} Y_{23}-X_{23} Y_{2}=-(-1)^{\partial X \partial Y} \int\left[\theta^{1} \partial_{2} Y\right] X d \theta^{1} d \theta^{2} d \theta^{3},
\end{aligned}
$$




$$
\begin{aligned}
& \psi_{4}(X, Y)=X_{2} Y_{12}-X_{12} Y_{2}=-(-1)^{\partial X \partial Y} \int\left[\theta^{3} \partial_{2} Y\right] X d \theta^{1} d \theta^{2} d \theta^{3}, \\
& \psi_{5}(X, Y)=X_{3} Y_{31}-X_{31} Y_{3}=-(-1)^{\partial X \partial Y} \int\left[\theta^{2} \partial_{3} Y\right] X d \theta^{1} d \theta^{2} d \theta^{3}, \\
& \psi_{6}(X, Y)=X_{3} Y_{23}-X_{23} Y_{3}=-(-1)^{\partial X \partial Y} \int\left[\theta^{1} \partial_{3} Y\right] X d \theta^{1} d \theta^{2} d \theta^{3}
\end{aligned}
$$

For the last two cocycles, one can write them as two independent combinations of the form

$$
-(-1)^{\partial X \partial Y} \int\left[\left(\eta_{1} \theta^{1} \partial_{1}+\eta_{2} \theta^{2} \partial_{2}+\eta_{3} \theta^{3} \partial_{3}\right) Y\right] X d \theta^{1} d \theta^{2} d \theta^{3}
$$

with $\eta_{1}+\eta_{2}+\eta_{3}=0$. This example shows that, as soon as $N>2$, it is not possible to canonically associated a basis of one-cocycles to a given basis in $G_{N}$.

\subsubsection{Cyclic Cocycles on $G_{N}$. General Results}

- Cyclic cocycles of degree 0 on $G_{N}$

Cyclic cocycles of degree 0 are one-forms on $G_{N}$ that have to satisfy the conditions $b \vartheta=0$ and $\lambda \vartheta=\vartheta$. At this order the second condition gives nothing and the first is trivially satisfied because $b \vartheta(X, Y)=X Y-(-1)^{\partial X \partial Y} Y X=0$, since the algebra is graded commutative. One can therefore always identify the space of cyclic cocycles of degree zero (and the group of cyclic cohomology of degree 0 ) with the dual of $G_{N}$. It is therefore of dimension $2^{N}$. A general cyclic cocyle of degree zero takes the form $\Omega_{P}(X)=\int d^{N} \theta P(\theta) X, P$ being a polynomial in $\theta$.

- Cyclic cocyles of degree 1 on $G_{N}$

Although the cyclic cohomology of Grassmann algebras has not been determined explicitly, the dimensionality of the groups of cyclic cohomology $H_{\lambda}^{p}\left(G_{N}\right)$ has been calculated [7] (thanks to a Künneth-like formula that we will discuss later). The results are the following. For $p=0$, the dimension is $2^{N}-$ this, we already know since the space of zero cocyles coincides with the vector space of one forms on $G_{N}$. For any $p$, the " $Z_{2}$-graded dimension" $d$ is the coefficient of $t^{p}$ in the expansion of $P(t)=\left[2^{N-1}(1+\varepsilon)-(1-t)^{N}\right] /\left[(1+t)(1-t)^{N}\right]$ where $\varepsilon$ is a $Z_{2}$ generator ${ }^{8}$. Actually, one can write $d$ as $d=d_{e}+d_{o} \varepsilon$, where $d_{e}$ (respectively $d_{o}$ ) is the number of even (respectively odd) cyclic cocycles of degree $p$. The total number of cyclic cocycles of degree $p$ is therefore $d_{t}=d_{e}+d_{o}$. For $N=2$ and $p=1$ the reader can check that $d=3+2 \varepsilon$ (i.e. three even generators and two odd for the one-cocycles), in accordance with the previous explicit results. An analogous situation holds for $N=3$, where one gets $d=9+8 \varepsilon$ one-cocycles. The number of independent one-cocycles grows rapidly with $N$; it is easy to show that one gets $(N-1) 2^{N-1}+1$ even one-cocycles and $(N-1) 2^{N-1}$ odd one-cocycles. Altogether, $d_{t}=(N-1) 2^{N}+1,(d=25+24 \varepsilon \approx 49$ for $N=4)$.

We have seen that for $N=1,2$ and 3 , the cyclic cocycles of degree one may be expressed as Berezin integrals $\omega_{\mathscr{D}}(X, Y)=(-1)^{\partial X \partial Y} \int[\mathscr{D} Y] X d^{N} \theta$, where $\mathscr{D}$ is a differential operator of degree one in the Grassmann algebra. This result can be extended for general $N$ as follows. We first take a bilinear form $\omega_{\mathscr{D}}$ from $G_{N} \times G_{N}$

${ }^{8}$ Actually, for $p$ even one has to add 1 to $d$ (see Sect. 2.1.7) 
in $R, \omega_{\mathscr{D}}(X, Y)=(-1)^{\partial X \partial Y} \int[\mathscr{D} Y] X d^{N} \theta$. We may assume that $\mathscr{D}=\sum_{i=1}^{N} P_{i}(\theta) \partial_{i}$, where $P_{i}(\theta)$ is a polynomial in the Grassmann variables $\theta$. Integrating by parts (taking care of graded commutation $\left.X Y=(-1)^{\partial X \partial Y} Y X\right)$ leads to the following expression:

$$
\omega_{\mathscr{D}}(X, Y)=(-1)^{1+\partial X \partial Y} \omega_{\mathscr{D}}(X, Y)-\int X\left(\operatorname{div}^{g} \mathscr{D}\right) Y d^{N} \theta
$$

where the graded divergence is defined by ${ }^{9}$

$$
\operatorname{div}^{g} \mathscr{D} \equiv \sum_{i=1}^{N} \partial_{i} P_{i}^{*} \equiv \sum_{i=1}^{N} \partial_{i} P_{i}^{+}-\partial_{i} P_{i}^{-} .
$$

Requiring the graded antisymmetry (Eq. (9)) implies:

$$
\operatorname{div}^{g} \mathscr{D}=0 .
$$

The cocycle condition is then trivially satisfied thanks to the divergence equation:

$$
\omega_{\mathscr{D}}(X Y, Z)+\omega_{\mathscr{D}}(Y Z, X)+\omega_{\mathscr{D}}(Z X, Y)=\sum_{i=1}^{N} \int \partial_{i}\left(P_{i}^{*} X Y Z\right) d^{N} \theta=0 .
$$

Therefore, $\omega_{\mathscr{D}}(X, Y)$ is a cyclic cocycle on $G_{N}$ provided the differential operator $\mathscr{D}$ is graded divergenceless: $\operatorname{div}^{g} \mathscr{D}=0$.

- Non-triviality and linear independence of the cyclic cocycles on $G_{N}$

One has to verify that $\omega_{\mathscr{D}}$ is not a trivial one-cocycle, i.e. a coboundary $b \Omega_{P}$, where $\Omega_{P}$ is a zero-cocycle:

$$
\Omega_{P}(X)=\int d^{N} \theta P(\theta) X
$$

$P$ being a polynomial in $\theta$. The coboundaries are of the form $\left[b \Omega_{P}\right](X, Y)=$ $\Omega_{P}([X, Y])$. As we have a graded commutative algebra, one has $[X, Y]=0 \forall X, Y$. Therefore the coboundaries vanish identically. Since $\mathscr{D} \neq 0$, we conclude that $\omega_{\mathscr{D}}$ is not trivial.

On the other hand, the differential operator $\mathscr{D}$ depends on $N 2^{N}$ coefficients. The cocycle condition $\operatorname{div}^{g} \mathscr{D}=0$, projected on each product of $\theta$ variables leads to $2^{N}-1$ non-coupled (and thus independent) equations of constraints (there are exactly $2^{N}-1$ equations since the $\theta^{1} \cdots \theta^{N}$ element cannot appear in $\operatorname{div}^{g} \mathscr{D}$, due to the action of the derivatives $\left.\partial_{i}\right)$. One obtains in this way $N 2^{N}-\left(2^{N}-1\right)=$ $(N-1) 2^{N}+1$ independent divergenceless differential operators $\mathscr{D}$.

Since each of these operators leads to a non-trivial one-cocycle, and that we have $(N-1) 2^{N}+1$ independent divergenceless differential operators $\mathscr{D}$, one obtains $(N-1) 2^{N}+1$ independent non-trivial cyclic one-cocycle on $G_{N}$. As we know this is exactly the number of independent non-trivial cyclic one-cocycle on $G_{N}$, we deduce that we have all the non-trivial one-cocycles. Thus, every non-trivial cyclic

\footnotetext{
${ }^{9}$ For any super-function $P$ given by $P^{+}+P^{-}$, where $\partial P^{+}=0$ and $\partial P^{-}=1$, one defines the star super-function by $P^{*}=P^{+}-P^{-}$
} 
one-cocycle on $G_{N}$ has the form

$$
\omega_{\mathscr{D}}(X, Y)=\sum_{i=1}^{N}(-1)^{\partial X \partial Y} \int\left[P_{i} \partial_{i} Y\right] X d^{N} \theta=(-1)^{\partial X \partial Y} \int[\mathscr{D} Y] X d^{N} \theta
$$

with $\operatorname{div}^{g} \mathscr{D}=0$.

Remark that if $\mathscr{D}$ and $\mathscr{D}^{\prime}$ are differential operators defining cyclic cocycles, $\left[\mathscr{D}, \mathscr{D}^{\prime}\right]$ is such that $\operatorname{div}^{g}\left[\mathscr{D}, \mathscr{D}^{\prime}\right]=\mathscr{D}\left(\operatorname{div}^{g} \mathscr{D}^{\prime}\right)-(-1)^{\partial \mathscr{D} \partial \mathscr{D}^{\prime}} \mathscr{D}^{\prime}\left(\operatorname{div}^{g} \mathscr{D}\right)=0$, so $\left[\mathscr{D}, \mathscr{D}^{\prime}\right]$ also defines a cyclic cocycle. The set of derivations $\mathscr{D}$ such that $\operatorname{div}^{g} \mathscr{D}=0$ is a Lie subalgebra of the algebra of differential operators of degree one in the Grassmann variables.

In general, the cocycle $\omega_{\mathscr{D}}$ may have non-zero values on any couples $(X, Y)$ whatever the $Z_{2}$-grading of $X$ and $Y$. It is interesting to know under which condition a cocycle $\omega_{\mathscr{D}}$ vanishes. The cases where $\omega_{\mathscr{D}}=0$ for $\partial X \neq \partial Y$ is of particular interest. Actually, since the integration over the $\theta$ 's selects only the $\theta^{1} \cdots \theta^{N}$ component in $(\mathscr{D} Y) X$, one obtains the following result (which is easy to check by choosing specific values for $X$ and $Y$ ): the cocycle $\omega_{\mathscr{D}}$ vanishes on couples $(X, Y)$ such that $\partial X \neq \partial Y$ (respectively $\partial X=\partial Y$ ) if and only if the differential operator $\mathscr{D}$ has the same grading $\partial \mathscr{D}$ as the parity of $N$ (respectively of $N+1$ ).

\subsubsection{The Cyclic Cohomology of the Super-Circle}

We now want to determine the cyclic cocycles of degree one on the super-circle, i.e. on the algebra $A_{N}=C\left(S^{1}\right) \otimes G_{N}$. Rather than trying to solve directly the equations of cyclicity and of vanishing of $b \Phi$ in this case, it is easier to use the knowledge that we have already on the pieces $G_{N}$ and $C\left(S^{1}\right)$ along with a theorem which allows us to compute the cyclic cohomology of the algebra $A \otimes B$ as soon as we know those of $A$ and of $B$. Such a theorem is proven in [10,11].

According to [12], the Hochschild cohomology of a product is given by the following relation: $H^{*}(A \otimes B)=H^{*}(A) \otimes H^{*}(B) .{ }^{10}$ The formula giving the cyclic cohomology groups $H_{\lambda}^{p}(A \otimes B)$ is slightly more involved [7]. The first result that we need (and which is rather easy to prove, cf. $[5,8]$ ) is that the cyclic cohomology of the algebra of complex numbers $H_{\lambda}^{p}(C)$ is 0 if $p$ is odd and equal to $C$ if $p$ is even. One can indeed prove that it is generated in degree two by the following form $\sigma(1,1,1)=2 i \pi$. Moreover, it is possible to show [5] that for any algebra $A$, $H_{\lambda}^{*}(A)$ is a module over $H_{\lambda}^{*}(C)$; indeed there is an operation (called "the operation $S$ of Connes") sending $H_{\lambda}^{p}(A)$ to $H_{\lambda}^{p+2}(A)$, and this action is fully characterized by the action of the generator $\sigma$. In order to prove the relevant formula, one has to assume that the algebra $B$ is such that $H_{\lambda}^{*}(B)$ can be written as

$$
H_{\lambda}^{*}(B)=H_{\lambda}^{*}(C) \otimes U^{*} \oplus V^{*},
$$

where $U^{*}=\bigoplus_{p \geqq 0} U^{p}$ is a finite dimensional vector space and where $V^{*}$ is a trivial module for the action of the operation $S$, i.e. $S=0$ on $V^{*}$. The only thing that we need here is that this property is indeed satisfied for the algebra $B=G_{N}$. Indeed, one can show that $H_{\lambda}^{*}\left(G_{N}\right)=H_{\lambda}^{*}(C) \oplus W^{*}$. The (graded) Poincaré polynomial of

10 The notation $H^{*}$ denotes the collection of $H^{p}$ 
the vector space $W^{*}$ is actually the quantity $P(t)$ that we introduced at the end of Sect. 2.1.6. For instance, in the case of $G_{1}$, we had $W^{0}=C$ and $W^{1}=C$ so that $H_{\lambda}=C \oplus C$ and $H_{\lambda}^{1}=C$. In the case of $G_{2}$, we had $W_{0}=C \oplus C \oplus C$ and $W_{1}=(C \oplus C \oplus C) \oplus(C \oplus C)$. More generally, $G_{N}$ has the required property, with $U^{0}=C, U^{i}=0$ for $i>0$ and $V^{*}=W^{*}$. For such an algebra $B$ one proves that

$$
H_{\lambda}^{n}(A \otimes B)=\bigoplus_{p+q=n}\left[H_{\lambda}^{p}(A) \otimes U^{q} \oplus H^{p}(A) \otimes V^{q}\right] .
$$

Notice that the second term involves Hochschild cohomology. In the present case, there are two substantial simplifications. The first comes from the fact that for a Grassmann algebra $U^{p}=0$ as soon as $p \geqq 1$. Let us assume that $A=C^{\infty}(\mathscr{M})$, where $\mathscr{M}$ is some finite dimensional smooth manifold. For one-cocyles, the formula reads

$$
H_{\lambda}^{1}\left(C^{\infty}(\mathscr{M}) \otimes G_{N}\right)=\Lambda_{0}(\mathscr{M}) \otimes W^{1}+H_{\lambda}^{1}\left(C^{\infty}(\mathscr{M})\right) \otimes C+\Lambda_{1}(\mathscr{M}) \otimes W^{0},
$$

where $\Lambda_{0}$ denotes the space of all currents of degree zero on $\mathscr{M}$ (distributions), $\Lambda_{1}$ denotes the space of currents of degree one on $\mathscr{M}$ and $H_{\lambda}^{1}\left(C^{\infty}(\mathscr{M})\right)$ coincides with the space of closed currents of degree one on $\mathscr{M}{ }^{11}$ The next simplification comes from taking $\mathscr{M}=S^{1}$; then the current of degree one correspond to the distributions on the circle and the closed currents to the constants.

From our separate study of the cyclic cohomology of the circle and of Grassmann algebras, we conclude that, in the case of the super-circle, $H_{\lambda}^{1}\left(A_{N}\right)$ is spanned by the following three kinds of cocycles:

$d=(N-1) 2^{N}+1$ cocycles of the kind $\alpha_{i}(t) \otimes \varphi_{i}$, where the $\alpha_{i}$ 's denote arbitrary functions on the circle (actually distributions) and the $\varphi_{i}$ 's are cyclic cocycles of degree one on $G_{N}$-which have the same $Z_{2}$-grading as the cocycles of $G_{N}$. The first class of cocycles corresponds to the first term of the general "Künneth-like" formula (27).

One cocycle of the kind $a_{1} \omega_{c} \otimes \vartheta_{1}$, where $a_{1}$ is a arbitrary constant, $\omega_{c}$ is the usual cyclic cocycle on the circle and $\vartheta_{1}$ is the cocycle of degree zero on $G_{N}$ defined as the one form vanishing on all the generators of $G_{N}$ but the unit. Notice that this cocycle is even. This second class of cocycles (containing only one element) corresponds to the second term of the general formula. ${ }^{12}$

Finally, we obtain $2^{N}-1$ cocycles of the kind $a_{k}(t) \omega_{c} \otimes \vartheta_{k}$, where $a_{k}(t)$ are arbitrary distributions, $\omega_{c}$ is the usual cocycle on $C\left(S^{1}\right)$ and $\vartheta_{k}$ are cyclic cocycles of degree zero on $G_{N}$ - the special cocycle $k=1$ has already been singled out. It is clear that $2^{N-1}$ among those cocycles are odd and the others $\left(2^{N-1}-1\right)$ are even. This last class of cocycles corresponds to the last term of the general formula.

Altogether, we find that the cyclic cocycles of degree one on the super-circle $A_{N}$ span a vector space of dimension $N 2^{N}+1$. They depend upon $N 2^{N}$ arbitrary distributions and one constant.

Now, one wishes to associate to each cocycle a first order differential operator

${ }^{11}$ Here we make use of the result given in the footnote 7 of Sect. 2.1.4

12 The particular cocycle of degree zero on $G_{N}$ called $\vartheta_{1}$ in Sect. 2.1 .5 is actually rather special. Indeed, by repeated action of the operator $S$, it leads to a hierarchy of non-trivial cyclic cocycles of degree $2 n$. Repeated action of $S$ (stabilisation) on the other zero cocycles lead to cocycles that are cohomologically trivial. This is discussed in [13] along with several elementary properties of Grassmann algebras 
$\hat{\mathscr{D}}=Q_{0}\left(t, \theta^{1}, \cdots, \theta^{N}\right) \hat{\partial}_{t}+\sum_{i=1}^{N} Q_{i}\left(t, \theta^{1}, \cdots, \theta^{N}\right) \partial_{i}$ (let us recall that the notation $\hat{\partial}_{t}$ means $\left.\hat{\partial}_{t}=t \partial_{t}\right)$ in such a way the cocycle reads ${ }^{13}$

$$
\omega_{\hat{\mathscr{D}}}(X, Y)=(-1)^{\partial X \partial Y} \int_{A_{N}}[\hat{\mathscr{D}} Y] X \frac{d t}{t} d^{N} \theta
$$

That seems to be a natural attempt in regard to the case $C^{\infty}\left(S^{1}\right) \otimes \mathscr{G}$ (i.e. Kac-Moody algebras) and $G_{N}$ considered before. The graded antisymmetry of the cocycle (28) again implies

$$
\operatorname{div}^{g} \hat{\mathscr{D}}=\hat{\partial}_{t} Q_{0}+\sum_{i=1}^{N} \partial_{i} Q_{i}^{*}=0
$$

and insures the cocycle condition to be satisfied. The non triviality of such a cocycle comes from the vanishing of the one-cyclic coboundaries on a graded commutative algebra (see Sect. 2.1.6).

In order to prove that each cocycle can be represented by (28) with condition (29), we just have to check that the linear vector space generated by the operators $\mathscr{D}$ has the same dimension as $H_{\lambda}^{1}\left(C^{\infty}\left(S^{1}\right) \otimes G_{N}\right)$. The most general differential operator is written with the help of $(N+1) 2^{N}$ distributions on $S^{1}$. The divergenceless equation leads to $2^{N}$ constraint equations on these distributions. From the vanishing of the $\theta$-highest degree term, we deduce that the coefficient of $\theta^{1} \ldots \theta^{N}$ in $Q_{0}\left(t, \theta^{1}, \cdots, \theta^{N}\right)$ is a constant. The remaining $2^{N}-1$ (non-coupled) equations involve the $(N+1) 2^{N}-1$ other distributions, and we are left with $N 2^{N}$ independent distributions, in accordance with the Künneth formula (27).

In the following, we will concentrate our attention to regular distributions associated to functions on $\mathrm{S}^{1}$.

\subsection{Explicit Results}

For the convenience of the reader, and because we will need them later, we gather the results for $N=1,2$ and 3 .

- The case $N=1$

According to the results of section 2.1.7, the most general one-cocycles are linear combinations of $a_{1} \omega_{c} \otimes \vartheta_{1}, a_{\theta} \omega_{c} \otimes \vartheta_{\theta}$ and $\alpha \otimes \varphi$, where $a_{1}$ is a constant, $a_{\theta}$ and $\alpha$ are functions of $t$ and $\omega_{c}(X, Y)$ is given by (6). We find it more convenient to express the results in terms of Berezin integrals and use the characterization of cyclic cocycles by the graded divergenceless differential operators described previously (we shall continue to adopt this attitude in the following). An arbitrary differential operator of order one on $A_{1}$ can be written as:

$$
\hat{\mathscr{D}}=a_{0} \hat{\partial}_{t}+a_{1} \theta \hat{\partial}_{t}+\varepsilon \partial_{\theta}+\sigma \theta \partial_{\theta} .
$$

Operators characterizing cyclic cocycles have to satisfy a divergence equation (29)

13 We use the symbol $\int_{A_{N}} \frac{d t}{t} d \theta^{1} \cdots d \theta^{N}$ for $\int_{|t|=1} \frac{d t}{t} \times$ Berezin integral over $\theta^{1} \cdots \theta^{N}$ 
which implies that $\hat{\partial}_{t} a_{0}=\sigma$ and $\hat{\partial}_{t} a_{1}=0$. This is of course in accordance with the results of Sect. 2.1.7. The $N=1$ cocycle takes the form

$$
\Phi_{N=1}(X, Y)=(-1)^{\partial X \partial Y} \frac{1}{2 i \pi} \int_{A_{1}}\left[\left(a_{0} \hat{\partial}_{t}+a_{1} \theta \hat{\partial}_{t}+\varepsilon \partial_{\theta}+\left(\hat{\partial}_{t} a_{0}\right) \theta \partial_{\theta}\right) Y\right] X \frac{d t}{t} d \theta
$$

which depends on one constant $a_{1}$ and two functions $a_{0}$ and $\varepsilon$ of $t$.

- The case $N=2$

The most general differential operator of order one of $A_{2}$ is:

$$
\widehat{\mathscr{D}}=a_{0} \hat{\partial}_{t}+a_{12} \theta^{1} \theta^{2} \hat{\partial}_{t}+\sum_{i}\left(a_{i} \theta^{i} \hat{\partial}_{t}+\varepsilon_{i} \partial_{i}+\lambda_{i} \theta^{1} \theta^{2} \partial_{i}\right)+\sum_{i j} \sigma_{i j} \theta^{i} \partial_{j}
$$

The divergence equation implies

$$
\hat{\partial}_{t} a_{0}=\sigma_{11}+\sigma_{22}, \quad \hat{\partial}_{t} a_{1}=\lambda_{2}, \quad \hat{\partial}_{t} a_{2}=-\lambda_{1}, \quad \hat{\partial}_{t} a_{12}=0 .
$$

Therefore the $N=2$ one-cocycle has the following form:

$$
\begin{aligned}
\Phi_{N=2}(X, Y)= & (-1)^{\partial X \partial Y} \frac{1}{2 i \pi} \int_{A_{2}}\left[\left(a_{0} \hat{\partial}_{t}+a_{12} \theta^{1} \theta^{2} \hat{\partial}_{t}+\hat{\partial}_{t} a_{1} \theta^{1} \theta^{2} \partial_{2}-\hat{\partial}_{t} a_{2} \theta^{1} \theta^{2} \partial_{1}\right.\right. \\
& \left.\left.+\sum_{i}\left(a_{i} \theta^{i} \hat{\partial}_{t}+\varepsilon_{i} \partial_{i}\right)+\sum_{i j} \sigma_{i j} \theta^{i} \partial_{j}\right) Y\right] X \frac{d t}{t} d \theta^{1} d \theta^{2}
\end{aligned}
$$

where $a_{12}$ is a constant and the other coefficients are functions of $t$.

- The case $N=3$

The most general differential operator of order one of $A_{3}$ is:

$$
\begin{aligned}
\hat{\mathscr{D}}= & a_{0} \hat{\partial}_{t}+a_{123} \theta^{1} \theta^{2} \theta^{3} \hat{\partial}_{t}+\sum_{i}\left(a_{i} \theta^{i} \hat{\partial}_{t}+\varepsilon_{i} \partial_{i}+\lambda_{i} \theta^{1} \theta^{2} \theta^{3} \partial_{i}\right) \\
& +\sum_{i j} \sigma_{i j} \theta^{i} \partial_{j}+\sum_{\operatorname{cyclic}(i, j, k)}\left(b_{k} \theta^{i} \theta^{j} \hat{\partial}_{t}+\sum_{m} \omega_{m k} \theta^{i} \theta^{j} \partial_{m}\right) .
\end{aligned}
$$

The notation cyclic $(i, j, k)$ means that we sum over cyclic permutations of $1,2,3$. The divergenceless condition leads to the following constraints on the coefficients:

$$
\hat{\partial}_{t} a_{0}=\sum_{m=1}^{3} \sigma_{m m}, \quad \hat{\partial}_{t} b_{i}=\lambda_{i}, \quad \hat{\partial}_{t} a_{123}=0, \quad \omega_{i j}-\omega_{j i}=\hat{\partial}_{t} a_{k}
$$

where $i, j, k$ is a cyclic permutation of $(1,2,3)$.

One obtains therefore for the $N=3$ one-cocycle:

$$
\begin{aligned}
\Phi_{N=3}(X, Y)= & (-1)^{\partial \mathrm{X} \partial Y} \frac{1}{2 i \pi} \int_{A_{3}}\left[\left(a_{0} \hat{\partial}_{t}+a_{123} \theta^{1} \theta^{2} \theta^{3} \hat{\partial}_{t}+\sum_{i}\left(a_{i} \theta^{i} \hat{\partial}_{t}+\varepsilon_{i} \partial_{i}+\hat{\partial}_{t} b_{i} \theta^{1} \theta^{2} \theta^{3} \partial_{i}\right)\right.\right. \\
& \left.\left.+\sum_{i j} \sigma_{i j} \theta^{i} \partial_{j}+\sum_{\operatorname{cyclic}(i, j, k)}\left(b_{k} \theta^{i} \theta^{j} \hat{\partial}_{t}+\sum_{m} \omega_{m k} \theta^{i} \theta^{j} \partial_{m}\right)\right) Y\right] X \frac{d t}{t} d \theta^{1} d \theta^{2} d \theta^{3},
\end{aligned}
$$

where $a_{123}$ is a constant and the other coefficients are functions of $t$. 


\section{Algebras of Super-Derivations (The General Framework)}

\subsection{General Discussion}

3.1.1. Derivations and Super-Derivations. Let $\operatorname{Der}\left(A_{0}\right)$ be the algebra of vector fields on the circle, it is also the algebra of derivations of $A_{0}=C\left(S^{1}\right)$. In the case $N \neq 0$, we will call $\operatorname{Der}\left(A_{N}\right)$ the algebra of graded derivations of $A_{N}=C\left(S^{1}\right) \otimes G_{N}$. It is clear that $\operatorname{Der}\left(A_{N}\right)$ is generated by derivations of the type $\mathscr{L}=V^{t} \partial_{t}+V^{\alpha} \partial_{\alpha}$, where $\partial_{t}=\partial / \partial t$ and $\partial_{\alpha}=\partial / \partial \theta^{\alpha}$. This notation can be condensed as $\mathscr{L}=V^{I} \partial_{I}$, where $I \in\{0,1,2, \ldots, N\}$ and where we set $V^{t}=V^{0}, \partial_{t}=\partial_{0}$. The quantities $V^{I}$ are, of course, functions on the super-circle, $V^{I}=V^{I}(t, \theta)=V_{J}^{I}(t) \theta^{J}$, where $J \in\left\{1, \ldots, 2^{N}\right\}$ and $\theta^{J}$ is a monomial element of $G_{N}$. Therefore $V_{J}^{I}(t)$ is a rectangular matrix of dimension $2^{N}(N+1)$ whose elements are functions of $t \in S^{1}$. Notice that for an arbitrary algebra $A$, the algebra of its derivations $\operatorname{Der} A$ is usually not a module over $A$ (i.e. if $a \in A$ and $\delta \in \operatorname{Der}(A)$, then $a \times \delta$ is usually not in $\operatorname{Der}(A))$. However, when $A$ is commutative of graded-commutative (as here), it is so. Indeed, $\operatorname{Der}\left(A_{N}\right)$ is a module of dimension $N+1$ over $A_{N}$ (and of dimension $2^{N}(N+1)$ over $C\left(S^{1}\right)$ ).

We will now build central extensions of super-loop algebras and examine what are the derivations compatible with a given extension.

3.1.2. The Compatibility Equation. If $\omega$ is a Lie algebra cocycle on the super-loop algebra $K_{N}$, one can build a central extension (cf. Eq. (2)) $K_{N, \omega}=K_{N} \oplus R \times e$, where $e$ is a new (central) generator and with a new Lie product, $[X, Y]_{\text {new }}=$ $[X, Y]_{\text {old }}+\omega(X, Y) \times e$. It is clear that not any (graded) derivation of $K_{N}$ will be a graded derivation of $K_{N, \omega}$. Such a graded derivation $\mathscr{L}$ should satisfy the property

$$
\mathscr{L}([X, Y])=[\mathscr{L} X, Y]+(-1)^{\partial X \partial \mathscr{L}}[X, \mathscr{L} Y],
$$

where $\partial \mathscr{L}$ is zero if it is a true derivation and one if it is antiderivation. From the above definition of $[,]_{\text {new }}$, it is clear that $\mathscr{L}$ will be a derivation if

$$
\omega(\mathscr{L} X, Y)+(-1)^{\partial X \partial \mathscr{L}} \omega(X, \mathscr{L} Y)=0 \text { for any } X, Y \in K_{N} .
$$

As described in Sect. 2, $K_{N}$ is obtained from $A_{N}$ by tensorization by matrices; in the same way, the Lie algebra cocycle $\omega$ can be gotten from a cyclic cocycle $\Phi$ on the super-circle $A_{N}$. In order to remove unnecessary complications, we can work with $A_{N}$ rather than $K_{N}$ : determining the Lie algebra of super-derivations of $K_{N, \omega}$ compatible with a given Lie algebra cocycle $\omega$ amounts to determining the super-derivations $\mathscr{L}$ of $A_{N}$ compatible with a cyclic cocycle $\Phi$ of $A_{N}$, i.e. it amounts to selecting a subalgebra $\operatorname{Der}_{\Phi}\left(A_{N}\right)$ of $\operatorname{Der}\left(A_{N}\right)$ characterized by the $\mathscr{L}$ such that

$$
\Phi(\mathscr{L} X, Y)+(-1)^{\partial X \partial \mathscr{L}} \Phi(X, \mathscr{L} Y)=0 \text { for any } X, Y \in A_{N} \text {. }
$$

If we write $\Phi(X, Y)$ as $(-1)^{\partial X \partial Y} \frac{1}{2 i \pi} \int_{A}(\hat{\mathscr{D}} Y) X \frac{d t}{t} d^{N} \theta$, where $\hat{\mathscr{D}}$ is a differential operator of first order (we saw that it is always possible), the previous equation simply reads (using $\Phi(X, \mathscr{L} Y)=(-)^{1+\partial X(\partial \mathscr{L}+\partial Y)} \Phi(\mathscr{L} Y, X)$ and integrating by part)

$$
[\mathscr{L}, \mathscr{D}]+\left(\operatorname{div}^{g} \mathscr{L}\right) \mathscr{D}=0,
$$

where $\hat{\mathscr{D}}$ and $\mathscr{D}$ are related by $\hat{\mathscr{D}}=t \mathscr{D}$ and the definition of $\operatorname{div}^{g}$ given in (24) is now extended to the $N+1$ variables $t, \theta^{1}, \ldots, \theta^{N}$ of $A_{N}$. 
Notice that if $\Phi$ is associated to a distribution, this equation has to be understood in the sense of the distribution, i.e. applied to any test functions $X$ and $Y$ :

$$
\frac{1}{2 i \pi} \int_{A_{N}}\left[\left([\mathscr{L}, \mathscr{D}]+\left(\operatorname{div}^{g} \mathscr{L}\right) \mathscr{D}\right) Y\right] X \frac{d t}{t} d N \theta=0 \text { for any } X, Y \in A_{N} .
$$

A derivation $\mathscr{L}$ which satisfies such an equation will be called a derivation compatible with the cyclic cocycle $\Phi$ (given by the differential operator $\mathscr{D}$ ) or D-compatible.

This formula suggests a few comments. First, using the relation

$$
\operatorname{div}^{g}\left[\mathscr{L}, \mathscr{L}^{\prime}\right]=\mathscr{L}\left(\operatorname{div}^{g} \mathscr{L}^{\prime}\right)-(-1)^{\partial \mathscr{L} \partial \mathscr{L}^{\prime}} \mathscr{L}^{\prime}\left(\operatorname{div}^{g} \mathscr{L}\right)
$$

the compatibility equation for some $\mathscr{L}$ and some $\mathscr{L}^{\prime}$ implies the compatibility equation for $\left[\mathscr{L}, \mathscr{L}^{\prime}\right]$ :

$$
\left(\operatorname{div}^{g}\left[\mathscr{L}, \mathscr{L}^{\prime}\right]\right) \mathscr{D}+\left[\left[\mathscr{L}, \mathscr{L}^{\prime}\right], \mathscr{D}\right]=0 .
$$

In other words, the subset of $\mathscr{D}$-compatible super-derivations $\mathscr{L}$ is a graded Lie subalgebra of the graded Lie algebra of super-derivations. Secondly, since $\operatorname{div}^{g} \mathscr{D}=0$, the differential operator $\mathscr{D}$ defining the cocycle $\omega_{\mathscr{D}}$ is itself a $\mathscr{D}$ compatible super-derivation if $\mathscr{D}$ is even (whilst if $\mathscr{D}$ is odd $[\mathscr{D}, \mathscr{D}]$ represents an anticommutator which does not vanish necessarily). Finally, we notice that the compatibility equation represents actually two sets of equations since we demand the super-derivation $V$ to be of definite $Z_{2}$-grading.

Thus, for a given cocycle $\Phi-$ a given differential operator $\mathscr{D}-$, the previous compatibility equation selects a sub-algebra $\operatorname{Der}_{\Phi}\left(A_{N}\right)$ of the algebra of all derivations. This sub-algebra is the algebra of derivations of the extension $A_{N}, \Phi$. Notice that these algebras $\operatorname{Der}_{\Phi}\left(A_{N}\right)$ are also graded Lie algebras and could themselves possess non-trivial extensions: we do not study here these possible extensions of these algebras of derivations.

3.1.3. A Problem of Stratification. An arbitrary cocycle of $A_{N}$ is a priori a linear combination of $(N-1) 2^{N}+1+2^{N}$ generators, with coefficients which are either constants or functions on the circle (cf. the previous section). Therefore, the solution of the compatibility equations (for given $N$ ) will a priori depend upon the constants (or the functions) entering the expression of the cocycle. It is already clear that the structure of the algebra $\operatorname{Der}_{\Phi}\left(A_{N}\right)$ will depend on the vanishing or not of these coefficients. We shall say that two cocycles $\Phi_{1}$ and $\Phi_{2}$ are in the same stratum if they lead to the same (or isomorphic) subalgebras $\operatorname{Der}_{\Phi_{i}}\left(A_{N}\right)$. The problem consists of determining all the strata in the vector space of cocycles along with the partial ordered set of subalgebras corresponding to these different strata. We will solve the case $N=1$ and give partial results on $N=2,3$.

\subsection{Study of the Case $N=1, N=2, N=3$}

3.2.1. The Case with One Grassmann Generator. As usual, for any function $f(t)$ defined on the circle $S^{1}$, one introduces its Fourier modes by the expression $f(t)=\sum_{m \in Z} f_{m} t^{-m}$. The super-algebra $K_{1}$ built from a simple Lie algebra $\mathscr{K}$ with 
generators $T_{a}$, where $a=1, \ldots, \operatorname{dim} \mathscr{K}$ will be spanned by $T_{a}^{m}(t, \theta)=T_{a} t^{m}$ and $\Theta_{a}^{m}(t, \theta)=T_{a} t^{m} \theta$, where $m \in \mathbf{Z}$, satisfying the commutation relations:

$$
\begin{aligned}
{\left[T_{a}^{m}, T_{b}^{n}\right] } & =f_{a b}^{c} T_{c}^{m+n}, \\
{\left[T_{a}^{m}, \Theta_{b}^{n}\right] } & =f_{a b}^{c} \Theta_{c}^{m+n}, \\
\left\{\Theta_{a}^{m}, \Theta_{b}^{n}\right\} & =0 .
\end{aligned}
$$

The super-Kač-Moody algebra obtained from the $N=1$ cocycle given by (31) has the following non-trivial commutation relations:

$$
\begin{aligned}
{\left[T_{a}^{m}, T_{b}^{n}\right] } & =f_{a b}^{c} T_{c}^{m+n}+a_{1} m \delta_{m+n, 0} \delta_{a b}, \\
{\left[T_{a}^{m}, \Theta_{b}^{n}\right] } & =f_{a b}^{c} \Theta_{c}^{m+n}+a_{0 m+n} \delta_{a b}, \\
\left\{\Theta_{a}^{m}, \Theta_{b}^{n}\right\} & =-\varepsilon_{m+n} \delta_{a b},
\end{aligned}
$$

where we used the mode expansion of $\varepsilon(t)$ and $a_{0}(t)$.

The most general even super-derivation is

$$
\mathscr{L}_{\text {even }}=A(t) \partial_{t}+D(t) \theta \partial_{\theta}
$$

and the most general odd super-derivation is

$$
\mathscr{L}_{\text {odd }}=C(t) \theta \partial_{t}+B(t) \partial_{\theta} .
$$

The compatibility equations have the form

$$
\begin{gathered}
\left(\partial_{t} a_{0}\right) A-a_{0} D=0, \\
\partial_{t}(\varepsilon A)-2 \varepsilon D=0, \\
\left(\partial_{t} a_{0}\right) B-a_{0}\left(\partial_{t} B\right)=0, \\
\varepsilon C+a_{1} B=0,
\end{gathered}
$$

which lead to different super-derivation algebras according to the vanishing or not of the one-cocycle coefficients. One obtains explicitly: ${ }^{14}$

- $\operatorname{Der}\left(A_{1}, a_{0}\right)$

The solutions of the compatibility equations are $D=\frac{\partial a_{0}}{a_{0}} A$ and $B=c a_{0}$, where $c$ is a complex constant, and therefore the super-derivation algebra is given by

$$
\mathscr{L}=A\left(\partial_{t}+\frac{\partial a_{0}}{a_{0}} \theta \partial_{\theta}\right)+C \theta \partial_{t}+c a_{0} \partial_{\theta},
$$

where $c$ is a complex constant.

Taking for the super-derivations a basis $L_{n}=-t^{n+1} \partial_{t}-\frac{\partial_{t} a_{0}}{a_{0}} t^{n+1} \theta \partial_{\theta}, C_{n}=$ $\frac{1}{a_{0}} t^{n+1} \theta \partial_{t}$ and $B=a_{0} \partial_{\theta}$ (assuming of course that $a_{0}$ does not vanish for any $t,|t|=1$ ),

\footnotetext{
14 The notation $\operatorname{Der}\left(A_{1}, c_{1}, c_{2}, \ldots, f_{1}, f_{2}, \ldots\right)$, where $c_{i}$ are constants and $f_{i}$ functions of $t$, means that we choose a cocycle $\Phi$ of the kind (31) with all coefficients set to zero except those explicitly written. Note that the functions $f_{i}$ are not allowed to vanish on the circle $S^{1}$. The corresponding super-Kač-Moody algebra is given by Eq. (43), the non-vanishing central extension terms being those where $c_{i}$ and $f_{i}$ appear
} 
we find the following commutation relations:

$$
\begin{array}{lll}
{\left[L_{n}, L_{m}\right]=(n-m) L_{n+m}} & \left\{C_{n}, C_{m}\right\}=0 & {\left[L_{n}, B\right]=0} \\
{\left[L_{n}, C_{m}\right]=(n-m) C_{n+m}} & \left\{C_{n}, B\right\}=-L_{n}
\end{array}
$$

- $\operatorname{Der}\left(A_{1}, a_{1}\right)$

The solution is now $B=0$ and one has

$$
\mathscr{L}=A \partial_{t}+D \theta \partial_{\theta}+C \theta \partial_{t} .
$$

Taking for the super-derivations a basis $L_{n}=-t^{n+1} \partial_{t}, C_{n}=t^{n+1} \theta \partial_{t}$ and $D_{n}=t^{n} \theta \partial_{\theta}$, we find the following commutation relations:

$$
\begin{array}{ll}
{\left[L_{n}, L_{m}\right]=(n-m) L_{n+m},} & {\left[L_{n}, C_{m}\right]=(n-m) C_{n+m},} \\
{\left[L_{n}, D_{m}\right]=-m D_{n+m},} & {\left[D_{n}, D_{m}\right]=0,} \\
{\left[D_{n}, C_{m}\right]=C_{n+m},} & \left\{C_{n}, C_{m}\right\}=0 .
\end{array}
$$

- $\operatorname{Der}\left(A_{1}, \varepsilon\right)$

The solutions are given by $D=\frac{1}{2 \varepsilon} \partial_{t}(\varepsilon A)$ and $C=0$. One obtains

$$
\mathscr{L}=A \partial_{t}+\frac{1}{2 \varepsilon} \partial_{t}(\varepsilon A) \theta \partial_{\theta}+B \partial_{\theta}
$$

The commutation relations between the generators $L(A)=A \partial_{t}+\frac{1}{2 \varepsilon} \partial_{t}(\varepsilon A) \theta \partial_{\theta}$ and $G(B)=\varepsilon B \partial_{\theta}$ are

$$
\begin{aligned}
& {\left[L\left(A_{1}\right), L\left(A_{2}\right)\right]=L\left(A_{1} \partial_{t} A_{2}-A_{2} \partial_{t} A_{1}\right)} \\
& {\left[L\left(A_{1}\right), G\left(B_{1}\right)\right]=G\left(A_{1} \partial\left(B_{1}\right)-\frac{B_{1}}{2 \varepsilon} \partial\left(\varepsilon A_{1}\right)\right),} \\
& \left\{G\left(B_{1}\right), G\left(B_{2}\right)\right\}=0 .
\end{aligned}
$$

In the case where $\varepsilon$ is a constant function, taking $L_{n}=-t^{n+1} \partial_{t}-\frac{n+1}{2} t^{n} \theta \partial_{\theta}$ and $G_{n}=t^{n} \partial_{\theta}$, Eq. (52) leads to the relations:

$$
\left[L_{n}, L_{m}\right]=(n-m) L_{n+m}, \quad\left[L_{n}, G_{m}\right]=\left(\frac{n+1}{2}-m\right) G_{n+m}, \quad\left\{G_{n}, G_{m}\right\}=0 .
$$

- $\operatorname{Der}\left(A_{1}, a_{0}, a_{1}\right)$

One has for the compatibility equations $B=0$ and $D=\frac{\partial a_{0}}{a_{0}} A$. One obtains the super-derivation algebra

$$
\mathscr{L}=A\left(\partial_{t}+\frac{\partial a_{0}}{a_{0}} \theta \partial_{\theta}\right)+C \theta \partial_{t} .
$$

The commutation relations are those of the super-derivation algebra $\operatorname{Der}\left(A_{1}, a_{0}\right)$ restricted to the generators $L_{n}$ and $C_{n}$.

- $\operatorname{Der}\left(A_{1}, a_{1}, \varepsilon\right)$ 
The solutions are $D=\frac{1}{2 \varepsilon} \partial_{t}(\varepsilon A)$ and $B=-\frac{\varepsilon}{a_{1}} C$. Thus the super-derivation
gebra is algebra is

$$
\mathscr{L}=A \partial_{t}+\frac{1}{2 \varepsilon} \partial_{t}(\varepsilon A) \theta \partial_{\theta}+C\left(\theta \partial_{t}-\frac{\varepsilon}{a_{1}} \partial_{\theta}\right)
$$

However, a redefinition of the $\theta$ parameter leads to the following expression:

$$
\mathscr{L}=A \partial_{t}+\frac{1}{2 \varepsilon} \partial_{t}(\varepsilon A) \theta \partial_{\theta}+C\left(\theta \partial_{t}-\varepsilon \partial_{\theta}\right)
$$

This super-derivation algebra is actually the $N=1$ super-Virasoro conformal algebra (also called Neveu-Schwarz-Ramond algebra) [1]. The commutation relations between the generators $L(A)=A \partial_{t}+\frac{1}{2 \varepsilon}\left(\partial_{t}(\varepsilon A)\right) \theta \partial_{\theta}$ and $G(C)=C\left(\theta \partial_{t}-\varepsilon \partial_{\theta}\right)$ are the following:

$$
\begin{aligned}
{\left[L\left(A_{1}\right), L\left(A_{2}\right)\right] } & =L\left(A_{1} \partial_{t} A_{2}-A_{2} \partial_{t} A_{1}\right), \\
{\left[L\left(A_{1}\right), G\left(C_{1}\right)\right] } & =G\left(A_{1} \partial_{t} C_{1}-C_{1} \partial_{t} A_{1}+\frac{C_{1}}{2 \varepsilon} \partial\left(\varepsilon A_{1}\right)\right), \\
\left\{G\left(C_{1}\right), G\left(C_{2}\right)\right\} & =L\left(-2 C_{1} C_{2}\right) .
\end{aligned}
$$

Taking $\varepsilon(t)=t^{2 \kappa-1}$ with $\kappa=0$ (Ramond sector) or $1 / 2$ (Neveu-Schwarz sector), $L_{n}=-t^{n+1} \partial_{t}-\frac{n}{2} t^{n} \theta \partial_{\theta}$ and $G_{n}=t^{n+1}\left(\theta \partial_{t}-t^{2 \kappa-1} \partial_{\theta}\right)$, Eq. (55) leads to the relations:

$$
\begin{aligned}
& {\left[L_{m}, L_{n}\right]=(m-n) L_{m+n},} \\
& {\left[L_{m}, G_{r}\right]=\left(\frac{m+2 \kappa}{2}-r\right) G_{m+r},} \\
& \left\{G_{r}, G_{s}\right\}=2 L_{r+s}
\end{aligned}
$$

with $m, n \in Z$ and, $r, s \in Z+\kappa$.

- $\operatorname{Der}\left(A_{1}, a_{0}, \varepsilon\right)$

The solutions are given by $A=c_{1} \frac{a_{0}^{2}}{\varepsilon}, B=c_{2} a_{0}, C=0$ and $D=c_{1} \frac{a_{0} \partial_{t} a_{0}}{\varepsilon}$. (The function $\varepsilon(t)$ is assumed not to vanish on the circle.) The super-derivation algebra is generated by

$$
\mathscr{L}=c_{1} \frac{a_{0}^{2}}{\varepsilon} \partial_{t}+c_{2} a_{0} \partial_{\theta}+c_{1} \frac{a_{0} \partial_{t} a_{0}}{\varepsilon} \theta \partial_{\theta},
$$

where $c_{1}, c_{2}$ are complex constants.

- $\operatorname{Der}\left(A_{1}, a_{0}, a_{1}, \varepsilon\right)$

The solutions are given by $A=c_{1} \frac{a_{0}^{2}}{\varepsilon}, B=c_{2} a_{0}, C=-\frac{a_{1}}{\varepsilon} B$ and $D=c_{1} \frac{a_{0} \partial_{t} a_{0}}{\varepsilon}$. (The function $\varepsilon(t)$ is assumed not to vanish the circle.) The super-derivation algebra is generated by

$$
\mathscr{L}=c_{1} \frac{a_{0}^{2}}{\varepsilon} \partial_{t}+c_{2} a_{0}\left(\partial_{\theta}-\frac{a_{1}}{\varepsilon} \theta \partial_{t}\right)+c_{1} \frac{a_{0} \partial_{t} a_{0}}{\varepsilon} \theta \partial_{\theta},
$$

where $c_{1}, c_{2}$ are complex constants. 
It might be worth to study in some detail among super-derivation algebras those which contain the Virasoro algebra. We note in particular that the algebras $\operatorname{Der}\left(A_{1}, a_{0}\right)$ and $\operatorname{Der}\left(A_{1}, a_{1}\right)$ admit an anticommuting conformal spin 2 generator $C_{m}$. The determination of the unitary representations of some of these algebras may be also interesting: since the upper limit for the discrete series of the unitary minimal models of the Virasoro algebra $c=1$ is pushed up in the case of the RNS algebra to the value $c=3 / 2$, one can expect a similar situation with these new algebras. In this last case, the possibility to associate to such an algebra a super-Kač-Moody one through a semi-direct sum and would then be particularly useful (for example for a coset construction).

3.2.2. The Case with Two Grassmann Generators. The most general even superderivation is

$$
\mathscr{L}_{\text {even }}=A \partial_{t}+B \theta^{1} \theta^{2} \partial_{t}+\sum_{i j} W_{i j} \theta^{i} \partial_{j}
$$

and the most general odd super-derivation is

$$
\mathscr{L}_{\text {odd }}=\sum_{i} L_{i} \theta^{i} \partial_{t}+C_{i} \partial_{i}+D_{i} \theta^{1} \theta^{2} \partial_{i} .
$$

The compatibility equations $N=2$ read (the indices $i, j$ run from 1 to 2 ):

$$
\begin{gathered}
\left(\partial a_{0}\right) A-a_{0}\left(W_{11}+W_{22}\right)=0, \\
\varepsilon_{1} B-\left(\partial a_{2}\right) A-a_{1} W_{21}+a_{2} W_{11}=0, \\
\varepsilon_{2} B+\left(\partial a_{1}\right) A-a_{1} W_{22}+a_{2} W_{12}=0, \\
\partial_{t}\left(\sigma_{i j} A\right)-a_{0} \partial_{t} W_{i j}+\sum_{m=1}^{2}\left(\sigma_{m j} W_{i m}-\sigma_{i m} W_{m j}-\sigma_{i j} W_{m m}\right)=0, \\
\partial_{t}\left(\varepsilon_{i} A\right)-\sum_{m=1}^{2}\left(\varepsilon_{i} W_{m m}+\varepsilon_{m} W_{m i}\right)=0, \\
\sum_{m=1}^{2}\left(\varepsilon_{m} L_{m}+a_{m} C_{m}\right)=0, \\
a_{12} C_{i}+a_{0} D_{i}-\sigma_{2 i} L_{1}+\sigma_{1 i} L_{2}=0, \\
a_{0} \partial_{t} C_{i}-\sum_{m=1}^{2} \sigma_{m i} C_{m}=0, \\
\partial_{t}\left(\varepsilon_{1} L_{1}\right)+a_{1} \partial_{t} C_{1}+C_{2} \partial_{t} a_{2}-\varepsilon_{1} D_{2}-\varepsilon_{2} D_{1}=0, \\
\partial_{t}\left(\varepsilon_{1} L_{2}\right)+a_{2} \partial_{t} C_{1}-C_{1} \partial_{t} a_{2}+2 \varepsilon_{1} D_{1}=0, \\
\partial_{t}\left(\varepsilon_{2} L_{1}\right)+a_{1} \partial_{t} C_{2}-C_{2} \partial_{t} a_{1}-2 \varepsilon_{2} D_{2}=0, \\
\partial_{t}\left(\varepsilon_{2} L_{2}\right)+a_{2} \partial_{t} C_{2}+C_{1} \partial_{t} a_{1}+\varepsilon_{2} D_{1}+\varepsilon_{1} D_{2}=0 .
\end{gathered}
$$

Since the cocycle $N=2$ contains 9 independent coefficients, the vanishing of some of these coefficients leads to $2^{9}=512$ different algebras, many of them being isomorphic. This gives an estimate of the large number of inequivalent strata. Although we have not performed a detailed analysis of all these cases, it is not too difficult to convince oneself that none of these algebras of super-derivations contains 
the $N=2$ superconformal algebra that will be defined in (80). Indeed, when inserting the explicit expression for the generators of the $N=2$ superconformal algebra in terms of super-derivations (cf. Eq. (80)) into the compatibility equations (61), one finds that the only solution is given by the zero cocycle: $\mathscr{D}=0$.

3.2.3. The Case with Three Grassmann Generators. The most general even superderivation is

$$
\mathscr{L}_{\text {even }}=A \partial_{t}+\sum_{i} K_{i} \theta^{1} \theta^{2} \theta^{3} \partial_{i}+\sum_{i j} H_{i j} \theta^{i} \partial_{j}+\sum_{\text {cyclic }(i, j, k)} D_{k} \theta^{i} \theta^{j} \partial_{t},
$$

and the most general odd super-derivation is

$$
\mathscr{L}_{\text {odd }}=B \theta^{1} \theta^{2} \theta^{3} \partial_{t}+\sum_{i}\left(C_{i} \theta^{i} \partial_{t}+E_{i} \partial_{i}\right)+\sum_{\operatorname{cyclic}(i, j, k)} \sum_{m} W_{m k} \theta^{i} \theta^{j} \partial_{m},
$$

where $\sum_{\text {cyclic }(i, j, k)}$ denotes a sum over the cyclic permutations of $(1,2,3)$.

The compatibility equations $N=3$ read (the indices $i, j$ runs from 1 to 3 in Eqs. (64.3) to (64.7), and the indices $i, j, k$ are circular permutations of $(1,2,3)$ in Eqs. (64.8) to (64.12), whilst $l=1,2,3$ in the three last equations:

$$
\begin{gathered}
\left(\partial a_{0}\right) A-a_{0}\left(H_{11}+H_{22}+H_{33}\right)=0 \\
\sum_{m=1}^{3}\left(a_{m} E_{m}+\varepsilon_{m} C_{m}\right)=0 \\
\left(\partial b_{i}\right) A-a_{0} K_{i}-\sum_{m=1}^{3} b_{m} H_{m i}+\sum_{m=1}^{3} \sigma_{m i} D_{m}=0 \\
\partial_{t}\left(\varepsilon_{i} A\right)-\sum_{m=1}^{3}\left(\varepsilon_{i} H_{m m}+\varepsilon_{m} H_{m i}\right)=0, \\
\sum_{m=1}^{3}\left(a_{m} W_{i m}+\omega_{i m} C_{m}\right)+a_{123} E_{i}+\varepsilon_{i} B=0, \\
a_{0} \partial_{t} E_{i}-\sum_{m=1}^{3} \sigma_{m i} E_{m}=0, \\
\partial_{t}\left(\sigma_{i j} A\right)-a_{0} \partial_{t} H_{i j}+\sum_{m=1}^{3}\left(\sigma_{m j} H_{i m}-\sigma_{i m} H_{m j}-\sigma_{i j} H_{m m}\right)=0, \\
\left(\partial a_{k}\right) A+\varepsilon_{i} D_{j}-\varepsilon_{j} D_{i}-a_{k}\left(H_{i i}+H_{j j}\right)+a_{i} H_{k i}+a_{j} H_{k j}=0, \\
\partial_{t}\left(\omega_{i l} A\right)+\partial_{t}\left(\varepsilon_{l} D_{i}\right)-\varepsilon_{l} K_{i}-\varepsilon_{i} K_{l}-a_{j} \partial_{t} H_{k l}+a_{k} \partial_{t} H_{j l}-\sum_{m=1}^{3}\left(\omega_{j m} H_{m i}+\omega_{i m} H_{m j}\right)=0, \\
\left(\partial a_{0}\right) C_{k}+a_{0}\left(W_{i j}-W_{j i}\right)+b_{j} E_{i}-b_{i} E_{j}-\sum_{m=1}^{3} \sigma_{k m} C_{m}=0, \\
\partial_{t}\left(\sigma_{k l} C_{j}-\sigma_{j l} C_{k}\right)-a_{0} \partial_{t} W_{l i}-b_{i} \partial_{t} E_{l}+E_{i} \partial_{t} b_{l}+\sum_{m=1}^{3}\left(\sigma_{m l} W_{i m}+\sigma_{m i} W_{l m}\right)=0 .(64) \\
\left(\partial_{t} \varepsilon_{l} C_{i}\right)+a_{i} \partial E_{l}+\varepsilon_{l} W_{j k}-\varepsilon_{j} W_{l k}-\varepsilon_{l} W_{k j}+\varepsilon_{k} W_{l j}+\omega_{l j} E_{k}-\omega_{l k} E_{j}=0,
\end{gathered}
$$

These equations lead to $2^{17}=131072$ different algebras, many of them being 
isomorphic. We will see in the next section that none of them contains the $N=3$ Ademollo et al. algebra, while only one contains the $N=2$ Ademollo et al. algebra.

\section{Superconformal Algebras and Algebras of Super-Derivations. Discussion and open Problems}

In string theory, the supersymmetric extension $(N=1)$ of the Virasoro algebra is known as the Ramond-Neveu-Schwarz (or RNS) algebra. A natural generalization to extended supersymmetries $(N>1)$ has been proposed by Ademollo et al. [2] a long time ago. The Virasoro algebra can be seen as the derivation algebra of the circle compatible with a Kač-Moody algebra. In the same way, the RNS algebra can be seen as a derivation algebra of the super-circle $A_{1}$ compatible with a non-trivial cocycle associated with a super-Kač-Moody algebra constructed from $A_{1}$ [1]. The situation changes drastically for $N \geqq 2$ : as we have seen by direct computation, there is no non-trivial cocycle compatible with the $N=2$ superconformal algebra. We will show below that this result holds for any $N$. However, it might seem reasonable, as for $N=0$ and $N=1$, and in the spirit of considering Kač-Moody algebras as ancestors of the super-Virasoro algebras, to associate for $N>1$ super-derivation algebras on extended super-circles with super-Kač-Moody algebras corresponding to non-trivial cocycles.

We will start this section by a rapid survey on the Ademollo et al. superconformal algebras (or ASC algebras), recalling the definitions and pointing out an interesting property which will be used in the study of the compatibility equation. Then in the light of this property, we present a construction of the $N=2$ ASC algebra. Finally we suggest two approaches leading to the construction of super-derivation algebras based on super-Kač-Moody algebras and generalizing the $N=1$ super-Virasoro algebra.

Note that our attempt to better understand the ASC algebras and to obtain a systematic classification of $N$-extended superconformal algebras is far from being the only one. At this point, it is worth mentioning, among different works, the recent approach of [14] based on supersymmetric $\sigma$ models on group manifolds, and the classification of [15] related to an underlying Clifford algebra structure in superconformal algebras.

\subsection{The Ademollo et al. Algebras}

4.1.1. Generalities. In the light of the RNS algebra, Ademollo et al. were able to combine in a consistent way conformal transformations with extended supersymmetric ones. These transformations can be reformulated as follows. In the $N$-extended superspace parameterized by the complex coordinates $\left(t, \theta^{1}, \ldots, \theta^{N}\right.$, $\left.\bar{t}, \bar{\theta}^{1}, \ldots, \bar{\theta}^{N}\right)$ with the metric $\delta_{i j}$ on the Grassmannian coordinates, we define as usual the covariant derivatives according to:

$$
D_{i}=\theta^{i} \partial_{t}+\partial_{i} \quad \text { with } \quad\left\{D_{i}, D_{j}\right\}=2 \delta_{i j} \partial_{t} \quad \text { and } \quad \partial_{i} \equiv \frac{\partial}{\partial \theta^{i}},
$$


and consider the super-analytic transformations

$$
\left(t, \theta^{1}, \ldots, \theta^{N}\right) \rightarrow\left(\tilde{t}, \widetilde{\theta}^{1}, \ldots, \widetilde{\theta}^{N}\right)
$$

which satisfy for all $i, j=1, \ldots, N$

$$
\bar{D}_{j} \tilde{t}=\bar{D}_{j} \tilde{\theta}^{i}=0 \quad \text { with } \quad \bar{D}_{i}=\bar{\theta}^{i} \partial_{\bar{t}}+\partial_{\bar{i}} \quad \text { and } \quad \partial_{\bar{i}} \equiv \frac{\partial}{\partial \bar{\theta}^{i}}
$$

(i.e. we restrict our attention to the $t, \theta^{1}, \ldots, \theta^{N}$ sector). Under such a transformation, the (super)covariant derivatives transform as:

$$
D_{i}=\left(D_{i} \tilde{\theta}^{j}\right) \tilde{D}_{j}+\left(D_{i} \tilde{t}-\tilde{\theta}^{j} D_{i} \tilde{\theta}^{j}\right) \tilde{\partial}_{t}
$$

Then the Ademollo et al. transformations can be seen as a super-analytic transformation satisfying

$$
D_{i} \tilde{t}=\tilde{\theta}^{j} D_{i} \tilde{\theta}^{j}
$$

i.e. the $D_{i}$ 's transform homogeneously as

$$
D_{i}=\left(D_{i} \tilde{\theta}^{j}\right) \tilde{D}_{j}
$$

At fixed $N$, the ASC algebra contains $2^{N}$ sets of generators, and among them can be recognized an $S O(N)$ Kač-Moody algebra. As examples, for $N=0$, we recover the Virasoro algebra generated by the $L_{m}(m \in Z)$ and for $N=1$, the RNS algebra generated by the $L_{m}(m \in Z)$ and the fermionic generators $G_{m},(m \in Z$ or $m \in Z+\frac{1}{2}$ ), while for $N=2$ an $S O(2)$ Kač-Moody algebra shows up. We also note the existence at order $N$ of the finite simple superalgebra $\operatorname{OSp}(N \mid 2)$ generated in the Neveu-Schwarz sector by $L_{ \pm 1,0} G_{ \pm 1 / 2}^{i}(i=1, \ldots, N)$ and the $T_{0}^{i j}=-T_{0}^{j i}=$ $\left\{G_{1 / 2}^{i}, G_{-1 / 2}^{j}\right\}$. Denoting by $\mathscr{L}=u \partial_{t}+\xi^{i} D_{i}$ the generators associated to an infinitesimal transformation given by Eq. (66), where $u=u\left(t, \theta^{1}, \ldots, \theta^{N}\right)$ and $\xi^{i}=\xi^{i}\left(t, \theta^{1}, \ldots, \theta^{N}\right)$ are super-functions, we deduce

$$
\left[\mathscr{L}, D_{i}\right]=\tilde{D}_{i}-D_{i}
$$

Now the condition (70) implies that, at the infinitesimal level, $\mathscr{L}$ acts linearly on the $D_{i}$ 's as

$$
\left[\mathscr{L}, D_{i}\right]=A_{i}^{j} D_{j}
$$

with (see footnote 9 for the definition of the star)

$$
\xi_{i}=\frac{1}{2} D_{i} u^{*} \quad \text { and } \quad A_{i}^{j}=D_{i} \xi^{j *}=-\frac{1}{2} D_{i} D_{j} u \quad(i=1, \ldots, N) .
$$

It is worthwhile to emphasize that the constraints (72), with $A_{i}^{j}$ to be determined, appear as a set of necessary and sufficient conditions for determining the ASC algebras.

4.1.2. The Ademollo et al. Algebras as Super-Derivation Algebras. We will determine in this section whether the $N$-ASC algebra may be viewed as a super-derivation algebra $\operatorname{Der}_{\Phi}\left(A_{N}\right)$ compatible with some cocycle $\Phi$. When looking at the defining relations (73) of the ASC algebras and (43) of the super-derivation algebras, it is tempting to connect these two approaches. Let us write these two relations:

$$
\left[\mathscr{L}, D_{i}\right]=-\frac{1}{2}\left(D_{i} D_{j} u\right) D_{j} \quad(i=1, \ldots, N) \quad \text { ASC algebras }
$$


with $\mathscr{L}=u \partial_{t}+\frac{1}{2}\left(D_{i} u^{*}\right) D_{i}$

$$
[\mathscr{L}, \mathscr{D}]=-\left(\operatorname{div}^{g} \mathscr{L}\right) \mathscr{D} \quad \text { super-derivation algebras. }
$$

One could think a priori that the $N$-ASC algebra is compatible with $N$ cocycles, each being a covariant derivative $D_{i}$. However, we will see that it is not the case. Actually, any cocycle $\mathscr{D}$ can be decomposed on the basis $\left\{\partial_{t}, D_{1}, \ldots, D_{N}\right\}$ as

$$
\mathscr{D}=\alpha_{0}(t, \theta) \partial_{t}+\sum_{i=1}^{N} \alpha_{i}(t, \theta) D_{i}
$$

with $\partial_{t} \alpha_{0}+\sum_{i=1}^{N} D_{i} \alpha_{i}^{*}=0$ (cf. Sect. 2.1.7).

The relation (43) leads then to two sets of equations, corresponding to the coefficient of $\partial_{t}$ and $D_{i}$ respectively:

$$
u \partial_{t} \alpha_{0}-\left(\partial_{t} u\right) \alpha_{0}-\frac{1}{2} \sum_{k=1}^{N}\left(D_{k} u^{*}\right)\left(D_{k} \alpha_{0}\right)=-(1-N / 2)\left(\partial_{t} u\right) \alpha_{0}
$$

$u \partial_{t} \alpha_{i}-\frac{1}{2}\left(\partial_{t} D_{i} u^{*}\right) \alpha_{0}^{*}-\frac{1}{2} \sum_{k=1}^{N}\left(\left(D_{k} u^{*}\right)\left(D_{k} \alpha_{i}\right)+\left(D_{k} D_{i} u\right) \alpha_{k}\right)=-(1-N / 2)\left(\partial_{t} u\right) \alpha_{i}$

In order to have the $N$-ASC algebra as a $\mathscr{D}$-compatible algebra, these equations have to be satisfied for any super-function $u$. Taking $u=1$ and $u=\theta^{i}(i=1, \ldots, N)$, one is led to

$$
\partial_{t} \alpha_{0}=\partial_{t} \alpha_{i}=0, \quad D_{k} \alpha_{0}=D_{k} \alpha_{i}=0 \quad \forall i, k .
$$

Then $u=t$ imposes $\alpha_{0}=0$ and $(N-1) \alpha_{i}=0 \forall i$.

Thus, for the cocycle $\mathscr{D}$ to be non-zero, one must have $N=1$. In this case, the cocycle $\mathscr{D}$ is given by the covariant derivative $D$, which is just the case found in Sect. 3.2.1 and in [1].

Therefore, the N-ASC algebras are super-derivation algebras compatible with some non-trivial cocycle $\mathscr{D}$ if and only if $N=1$.

4.1.3. The $N=2$ Case. Let us illustrate the above property (73) in the $N=2$ case. We set

$$
\mathscr{L}=u \partial_{t}+\xi^{1} D_{1}+\xi^{2} D_{2}
$$

and impose the conditions (72) for $i=1,2$. Then we deduce the following relations:

$$
\xi_{i}=\frac{1}{2} D_{i} u^{*}, \quad A_{i}^{j}=-\frac{1}{2} D_{i} D_{j} u .
$$

Using the decomposition $u=u_{0}+2 u_{i} \theta^{i}+2 u_{12} \theta^{1} \theta^{2}$, we find the generators of the $N=2$ ASC algebra:

$$
\begin{aligned}
L\left(u_{0}\right) & =u_{0} \partial_{t}+\frac{1}{2}\left(\partial_{t} u_{0}\right)\left(\theta^{1} \partial_{1}+\theta^{2} \partial_{2}\right), \\
G_{1}\left(u_{1}\right) & =u_{1}\left(\theta^{1} \partial_{t}-\partial_{1}\right)+\left(\partial_{t} u_{1}\right) \theta^{1} \theta^{2} \partial_{2}, \\
G_{2}\left(u_{2}\right) & =u_{2}\left(\theta^{2} \partial_{t}-\partial_{2}\right)-\left(\partial_{t} u_{2}\right) \theta^{1} \theta^{2} \partial_{1}, \\
T\left(u_{12}\right) & =u_{12}\left(\theta^{2} \partial_{1}-\theta^{1} \partial_{2}\right) .
\end{aligned}
$$


The commutation relations between these generators can be written as

$$
\begin{aligned}
& {\left[L\left(u_{0}\right), L\left(v_{0}\right)\right]=L\left(u_{0} \partial_{t} v_{0}-v_{0} \partial_{t} u_{0}\right), \quad\left[L\left(u_{0}\right), T\left(v_{12}\right)\right]=T\left(u_{0} \partial_{t} v_{12}\right),} \\
& {\left[L\left(u_{0}\right), G^{i}\left(v_{i}\right)\right]=G^{i}\left(u_{0} \partial_{t} v_{i}-\frac{1}{2} v_{i} \partial_{t} u_{0}\right), \quad\left[T\left(u_{12}\right), G^{i}\left(v_{i}\right)\right]=\varepsilon^{i j} G^{j}\left(u_{12} v_{i}\right),} \\
& \left\{G^{i}\left(u_{i}\right), G^{j}\left(v_{j}\right)\right\}=\delta^{i j} L\left(-2 u_{i} v_{j}\right)+\varepsilon^{i j} T\left(u_{i} \partial_{t} v_{j}-v_{j} \partial_{t} u_{i}\right) .
\end{aligned}
$$

Taking $L_{n}=-t^{n+1} \partial_{t}+\frac{n+1}{2} t^{n}\left(\theta^{1} \partial_{1}+\theta^{2} \partial_{2}\right), G_{r}^{1}=t^{r}\left(\theta^{1} \partial_{t}-\partial_{1}\right)+r t^{r-1} \theta^{1} \theta^{2} \partial_{1}, G_{r}^{2}=$ $t^{r}\left(\theta^{2} \partial_{t}-\partial_{2}\right)-r t^{r-1} \theta^{1} \theta^{2} \partial_{2}$ and $T_{n}=t^{n}\left(\theta^{2} \partial_{1}-\theta^{1} \partial_{2}\right)$, Eq. (78) leads to the relations:

$$
\begin{aligned}
& {\left[L_{m}, L_{n}\right]=(m-n) L_{m+n}, \quad\left[T_{m}, G_{r}^{i}\right]=\varepsilon^{i j} G_{m+r}^{j},} \\
& {\left[L_{m}, T_{n}\right]=-n T_{m+n}, \quad\left\{G_{r}^{i}, G_{s}^{j}\right\}=2 \delta^{i j} L_{r+s}+(r-s) \varepsilon^{i j} T_{r+s},} \\
& {\left[L_{m}, G_{r}^{i}\right]=(m / 2-r) G_{m+r}^{i},}
\end{aligned}
$$

$\varepsilon^{i j}$ being the antisymmetric tensor of order 2 . Here, one has $m, n \in Z$ and $r, s \in Z$ (Ramond sector) or $r, s \in Z+\frac{1}{2}$ (Neveu-Schwarz sector).

The $N=2$ two-dimensional superspace is a reducible representation of supersymmetry, which can be split into two parts, using the notion of $U(1)$-chirality. Defining $D$ and $\bar{D}$ as $D=\bar{\theta} \partial_{t}+2 \partial_{\theta}$ and $\bar{D}=\theta \partial_{t}+2 \partial_{\bar{\theta}}$, which $\theta=\theta^{1}+i \theta^{2}$ and $\bar{\theta}=\theta^{1}-i \theta^{2}$ (note that $\bar{\theta}$ has nothing to do with the $\bar{\theta}^{i}$ 's defined in Sect. 4.1.), a chiral (or antichiral) superfield $\Phi\left(t, \theta^{1}, \theta^{2}\right)$ satisfies $\bar{D} \Phi=0$ (or $D \Phi=0$ ).

Owing to Eq. (72) we will see that the $N=2$ ASC algebra is naturally related to this chirality. The $A$ matrix defined in Eq. (77) can be decomposed for any $N$ as $A=-\frac{1}{2}\left(\partial_{t} u\right) \mathbf{1}_{N}+\hat{A}$, with $\mathbf{1}_{N}$ being the $N \times N$ identity matrix and $\hat{A}$ an antisymmetric matrix. For $N=2$, we have

$$
A=-\frac{1}{2}\left(\partial_{t} u\right)\left(\begin{array}{ll}
1 & 0 \\
0 & 1
\end{array}\right)-\frac{1}{2} D_{1} D_{2} u\left(\begin{array}{cc}
0 & 1 \\
-1 & 0
\end{array}\right)
$$

which, after diagonalisation, leads to

$$
\begin{aligned}
& {[\mathscr{L}, D]=-\frac{1}{2}\left(\partial_{t} u-i D_{1} D_{2} u\right) D=-\frac{1}{4}(D \bar{D} u) D,} \\
& {[\mathscr{L}, \bar{D}]=-\frac{1}{2}\left(\partial_{t} u+i D_{1} D_{2} u\right) \bar{D}=-\frac{1}{4}(\bar{D} D u) \bar{D}}
\end{aligned}
$$

One easily deduces that (anti)chirality is preserved under the action of an $\mathscr{L}$ generator:

$$
D \Phi=0 \Rightarrow \mathscr{L} D \Phi=0 \quad \text { and } \quad \bar{D} \Phi=0 \Rightarrow \mathscr{L} \bar{D} \Phi=0 .
$$

Note that this last property can be viewed as a definition of the $N=2$ ASC algebra.

We already mentioned in Sect. 3.2. that there is no cocycle of a super-KačMoody algebra associated with a derivation algebra containing the $N=2$ ASC algebra. Even more, one can check that there is no chiral (antichiral) super-KačMoody algebra with a non-trivial cocycle whose derivation algebra contains the above algebra.

\subsection{New Kinds of Superconformal Algebras?}

As already mentioned in this paper, the Virasoro and the RNS algebras which are of particular physical interest are naturally related to (super)Kač-Moody algebras. 
At this point, it is tempting to try new kinds of $N>1$ superconformal algebras which would be algebras of super-derivations of some super-Kač-Moody ones. Hereafter, we will adopt two possible attitudes. First we will look for $N+1$ super-derivation algebras containing as a subalgebra the $N$ ASC algebra. Then in a completely different framework, we will propose a recurrent class of cocycles $\mathscr{D}_{N}$, with $\mathscr{D}_{1}$ being associated with the RNS algebra and $\mathscr{D}_{N+1}$ easily deduced from $\mathscr{D}_{N}$, and such that the corresponding derivation algebras are included into one another: $\operatorname{Der}_{\mathscr{D}_{N}}\left(A_{N}\right) \subset \operatorname{Der}_{\mathscr{D}_{N+1}}\left(A_{N+1}\right)$.

4.2.1. Embedding of the $N$-ASC Algebra in $\operatorname{Der}\left(A_{N+1}\right)$. Considering a super-loop algebra on $A_{N+1}$, we determine the possible cocycles $\omega_{\mathscr{D}}$ such that the $\mathscr{D}$-compatible super-derivation algebra contains the $N$-ASC algebra. The calculation is quite similar (although a little more tedious) to the previous one done in Sect. 4.1.2. Up to a rotation in the space of the $\theta$ variables, one can assume that the $N$-ASC algebra is described in terms of the $N$ first variables $\theta^{1}, \ldots, \theta^{N}$. We demand $\mathscr{D}$ to be such that $[\mathscr{L}, \mathscr{D}]=-\left(\operatorname{div}^{g} \mathscr{L}\right) \mathscr{D}$ with $\mathscr{D}=\alpha_{0} \partial_{t}+\sum_{i=1}^{N+1} \alpha_{i} D_{i}$ and $\mathscr{L}=u \partial_{t}+$ $\sum_{a=1}^{N}\left(D_{a} u^{*}\right) D_{a}$, where $a_{0}, a_{i} \in C^{\infty}\left(A_{N+1}\right)$ and $u \in C^{\infty}\left(A_{N}\right)$. One obtains

$$
\begin{gathered}
u \partial_{t} \alpha_{0}-\left(\partial_{t} u\right) \alpha_{0}+\sum_{a=1}^{N}\left(\frac{1}{2}\left(D_{a} u^{*}\right) D_{a} \alpha_{0}-\left(D_{a} u^{*}\right) \alpha_{a}^{*}\right)+\sum_{k=1}^{N+1}\left(D_{k} u^{*}\right) \alpha_{k}^{*}=\frac{N-2}{2}\left(\partial_{t} u\right) \alpha_{0} \\
u \partial_{t} \alpha_{a}-\frac{1}{2}\left(\partial_{t} D_{a} u^{*}\right) \alpha_{0}^{*}+\frac{1}{2} \sum_{k=1}^{N+1}\left(D_{k} D_{a} u^{*}\right) \alpha_{k}=\frac{N-2}{2}\left(\partial_{t} u\right) \alpha_{a} \\
u \partial_{t} \alpha_{N+1}+\frac{1}{2} \sum_{a=1}^{N}\left(D_{a} u\right) D_{a} \alpha_{N+1}=\frac{N-2}{2}\left(\partial_{t} u\right) \alpha_{N+1}
\end{gathered}
$$

Demanding these equations to be satisfied for any super-function $u$ (in order to have the $N$-ASC algebra), we obtain, taking $u=1, \theta^{a}, t$ with $a=1, \ldots, N$ and $j=1, \ldots, N+1$ :

$$
\begin{aligned}
& \partial_{t} \alpha_{0}=0, \quad D_{a} \alpha_{0}=0, \\
& \partial_{t} \alpha_{j}=0, \quad D_{a} \alpha_{N+1}=0, \quad \theta^{N+1} \alpha_{N+1}=-\frac{N}{2} \alpha_{0} . \\
& (N-2) \alpha_{N+1}=0, \quad(N-1) \alpha_{a}=0,
\end{aligned}
$$

These equations lead to the following results. The $N$-ASC algebra is embedded in a super-derivation algebra $\operatorname{Der}\left(A_{N+1}\right)$ compatible with some non-trivial cocycle $\mathscr{D}$ if and only if $N=1$ or $N=2$.

In the case of $N=1$, the cocycle is given by $\mathscr{D}=\left(c_{0}+c_{2} \theta^{2}\right)\left(\theta^{1} \partial_{t}+\partial_{1}\right)=$ $\left(c_{0}+c_{2} \theta^{2}\right) D_{1}$, where $c_{0}$ and $c_{2}$ are complex constants. This leads to three different super-derivation algebras.

In the case of $N=2$, there is only one (divergenceless) solution given by the cocycle $\mathscr{D}=c_{3} \partial_{3}$, where $c_{3}$ is a complex constant.

These algebras are displayed in the appendix. 
4.2.2. A Very Particular Chain of Cocycles and Their Corresponding Algebras. In this paragraph, we look for a chain of super-derivation algebras, each of them being compatible with a non-trivial cocycle, the $N^{\text {th }}$ order algebra being included into the $(N+1)^{\text {th }}$ order one, and such that for $N=1$, one recovers the RNS algebra.

When one looks at the super-derivations algebras $\operatorname{Der}_{\Phi}\left(A_{2}\right)$ containing the RNS algebra, two one-cocycles are singled out: $\mathscr{D}_{2}=D_{1}$ and $\mathscr{D}_{2}^{\prime}=\theta^{2} D_{1}$. The generalization of these cocycles is very easy: $\mathscr{D}_{N}=D_{1}$ and $\mathscr{D}_{N}^{\prime}=\theta^{N} \cdots \theta^{2} D_{1}$. We will study the two chains of super-derivation algebras, compatible with these two chains of cocycles.

- super-derivation algebra $\operatorname{Der}_{D_{1}}\left(A_{N}\right)$ The super-derivation $\mathscr{L}=w \partial_{t}+\sum_{i=1}^{N} \Psi_{i} D_{i}$ satisfies the compatibility equation
$\left[\mathscr{L}, D_{1}\right]=-\left(\operatorname{div}^{g} \mathscr{L}\right) D_{1}$. One has

$$
\left[\mathscr{L}, D_{1}\right]=\left(2 \Psi_{1}-D_{1} w^{*}\right) \partial_{t}-\sum_{i=1}^{N}\left(D_{1} \Psi_{i}^{*}\right) D_{i} \quad \text { and } \quad \operatorname{div}^{g} \mathscr{L}=\partial_{t} w+\sum_{i=1}^{N} D_{i} \Psi_{i}^{*}
$$

Therefore one obtains

$$
2 \Psi_{1}-D_{1} w^{*}=0 \quad \text { and } \quad D_{1} \Psi_{k}^{*}=\delta_{1 k}\left(\partial_{t} w-\sum_{i=1}^{N} D_{i} \Psi_{i}^{*}\right) \quad(k=1, \ldots, N) .
$$

Thus the super-derivation $\mathscr{L}$ has to satisfy

$$
\begin{aligned}
2 \Psi_{1}-D_{1} w^{*} & =0 \\
D_{1} \Psi_{n}^{*} & =0 \quad(n>1), \\
\sum_{n=2}^{N} D_{n} \Psi_{n}^{*} & =0
\end{aligned}
$$

Notice that the second equation implies that $\partial_{t} \Psi_{n}=0$ (for $n \neq 1$ ), i.e. the functions $\Psi_{n}$ are constant in $t$ for $n \neq 1$. We will call these generators "discrete generators." They commute with the Virasoro generators $L_{n}=-t^{n+1}-\frac{n+1}{2} t^{n} \theta^{1} \partial_{1}$ obtained for $w=-t^{n+1}$. Altogether this algebra contains $N 2^{N-1}+1$ generators among which $(N-2) 2^{N-1}+1$ "discrete" ones.

- super-derivation algebra $\operatorname{Der}_{\mathscr{D}_{N}^{\prime}}\left(A_{N}\right)$

A similar computation for the compatibility equation leads to the equations

$$
\begin{aligned}
\left(2 \Psi_{1}-D_{1} w^{*}\right) \theta^{N} \cdots \theta^{2} & =0 \\
D_{1}\left(\Psi_{n}^{*} \theta^{N} \cdots \theta^{2}\right) & =0 \quad(n>1), \\
\sum_{n=2}^{N} D_{n}\left(\Psi_{n}^{*} \theta^{N} \cdots \theta^{2}\right) & =0 .
\end{aligned}
$$

The last two equations are equivalent to the relations $\Psi_{n}^{0}=\Psi_{n}^{1}=0$ (with $n>1$ ) where $\Psi_{n}$ is decomposed as $\Psi_{n}(t, \theta)=\Psi_{n}^{0}(t)+\sum_{k=1}^{N} \Psi_{n}^{k}(t) \theta^{k}+\cdots$

This algebra contains $2^{N}+2 N\left(2^{N-1}-1\right)$ generators. If we don't worry about the discrete generators of $\operatorname{Der}_{D_{1}}\left(A_{N}\right)$, we see that the defining relation $2 \Psi_{1}-D_{1} w=0$ 
for $\operatorname{Der}_{D_{1}}\left(A_{N}\right)$ is stronger than the one of $\operatorname{Der}_{\mathscr{D}_{N}^{\prime}}\left(A_{N}\right)$. So we conclude that, up to the discrete generators, one has the inclusion

$$
\operatorname{Der}_{D_{1}}\left(A_{N}\right) \subset \operatorname{Der}_{\mathscr{D}_{N}^{\prime}}\left(A_{N}\right) \text {. }
$$

From the relations (73) and (74), one also has the embeddings (including the discrete generators)

$$
\begin{aligned}
\operatorname{Der}_{D_{1}}\left(A_{N}\right) \subset \operatorname{Der}_{D_{1}}\left(A_{N+1}\right), \\
\operatorname{Der}_{\mathscr{D}_{N}^{\prime}}\left(A_{N}\right) \subset \operatorname{Der}_{\mathscr{D}_{N+1}^{\prime}}\left(A_{N+1}\right) .
\end{aligned}
$$

Notice that the algebras $\operatorname{Der}_{D_{1}}\left(A_{2}\right)$ and $\operatorname{Der}_{\mathscr{D}_{2}^{\prime}}\left(A_{2}\right)$ have been given in Sect. 4.2.1 (for the commutation relations of these algebras, see the appendix).

\section{Conclusion}

In this paper, a classification of the possible central extensions for super-loop algebras defined on the super-circle $A_{N}$, where $N$ is the number of generators of the corresponding Grassmann algebra, has been achieved. A general method for determining the super-derivation algebra compatible with each super-Kač-Moody algebra has also been obtained, and made explicit in the cases $N=1,2,3$.

Surprisingly enough, the usual superconformal algebras for $N>1$ cannot be associated to super-Kač-Moody companions, contrarily to the case $N=0$ (Virasoro algebra) and $N=1$ (RNS algebra). Such a result led us to investigate for $N>1$ new types of extended superconformal algebras which contain the RNS algebra.

One can expect that some of these (super-Kač-Moody compatible) superderivation algebras which contain the Virasoro algebra, are well adapted to describe interesting two-dimensional conformal field theories. A study of these unitary representations is therefore necessary. The simplest examples to consider are, as already suggested at the end of Sect. 3.2.1, some of the algebras associated to the $N=1$ case. The property of such extended conformal algebras to be enlarged to a semi-direct sum by adjunction of a super affine algebra might be exploited. In particular new kinds of Wess-Zumino-Witten models [16] might be considered, generalizing the $N=1$ case treated in ref. [17].

\section{Appendix}

In this appendix, we will make explicit the commutation relations of some remarkable super-derivation algebras used in Sect. 4.2.

We recall that if $K$ is a loop-algebra with generators $T_{a}^{m}(m \in \mathbf{Z})$, constructed from a simple Lie algebra $\mathscr{K}$ with generators $T_{a}(a=1, \ldots, \operatorname{dim} \mathscr{K})$, one can associate a super-loop algebra $K_{N}$ with the generators $T_{a}^{m}=T_{a} t^{m},\left(\Theta^{i}\right)_{a}^{m}=T_{a} t^{m} \theta^{i}$, $\left(\Theta^{i j}\right)_{a}^{m}=T_{a} t^{m} \theta^{i} \theta^{j}, \ldots,\left(\Theta^{1 \cdots N}\right)_{a}^{m}=T_{a} t^{m} \theta^{1} \ldots \theta^{N}$. In the case $N=2$, the non-trivial commutation relations of the super-loop algebra take the explicit following form:

$$
\begin{aligned}
{\left[T_{a}^{m}, T_{b}^{n}\right] } & =f_{a b}^{c} T_{c}^{m+n}, & {\left[T_{a}^{m},\left(\Theta^{i}\right)_{b}^{n}\right] } & =f_{a b}^{c}\left(\Theta^{i}\right)_{c}^{m+n}, \\
{\left[T_{a}^{m},\left(\Theta^{12}\right)_{b}^{n}\right] } & =f_{a b}^{c}\left(\Theta^{12}\right)_{c}^{m+n}, & \left\{\left(\Theta^{1}\right)_{a}^{m},\left(\Theta^{2}\right)_{b}^{n}\right\} & =f_{a b}^{c}\left(\Theta^{12}\right)_{c}^{m+n} .
\end{aligned}
$$


For a super-derivation algebra, we will take for each independent generator $\phi(t) \partial_{I}$, where $\partial_{I}$ is a linear combination of $\partial_{t}$ and $\partial_{\theta_{I}}$, a basis $\phi_{m}=t^{m+d_{\phi}-1} \partial_{I}$, where the conformal weight $d_{\phi}$ of the generator $\phi(t) \partial_{I}$ is defined through the action of the Virasoro generator $L_{m}$ on it, i.e. $\left[L_{n}, \phi_{m}\right]=\left(n\left(d_{\phi}-1\right)-m\right) \phi_{n+m}$.

- super-derivation algebra $\operatorname{Der}_{\mathscr{D}}\left(A_{2}\right)$ with $\mathscr{D}=a_{2} \theta^{2}\left(\theta^{1} \partial_{t}+\partial_{1}\right)$

The super-Kač-Moody algebra obtained from this one-cocycle has the following non-trivial commutation relations:

$$
\begin{aligned}
{\left[T_{a}^{m}, T_{b}^{n}\right] } & =f_{a b}^{c} T_{c}^{m+n}-a_{2} m \delta_{m+n, 0} \delta_{a b}, & & {\left[T_{a}^{m},\left(\Theta^{i}\right)_{b}^{n}\right]=f_{a b}^{c}\left(\Theta^{i}\right)_{c}^{m+n} } \\
{\left[T_{a}^{m},\left(\Theta^{12}\right)_{b}^{n}\right] } & =f_{a b}^{c}\left(\Theta^{12}\right)_{c}^{m+n}, & & \left\{\left(\Theta^{1}\right)_{a}^{m},\left(\Theta^{2}\right)_{b}^{n}\right\}=f_{a b}^{c}\left(\Theta^{12}\right)_{c}^{m+n} \\
\left\{\left(\Theta^{1}\right)_{a}^{m},\left(\Theta^{1}\right)_{b}^{n}\right\} & =a_{2} \delta_{m+n, 0} \delta_{a b}, & &
\end{aligned}
$$

The super-derivations compatible with $\mathscr{D}$ are (after a redefinition of some generators)

$$
\begin{aligned}
\mathscr{L}_{\text {even }}= & A \partial_{t}+\frac{1}{2}(\partial A)\left(\theta^{1} \partial_{1}+\theta^{2} \partial_{2}\right)+T \theta^{2} \partial_{1}+W \theta^{2} \partial_{2}+B \theta^{1} \theta^{2} \partial_{t} \\
\mathscr{L}_{\text {odd }}= & G^{1}\left(\theta^{1} \partial_{t}-\partial_{1}\right)+\left(\partial G^{1}\right) \theta^{1} \theta^{2} \partial_{2} \\
& +G^{2} \theta^{2} \partial_{t}-\left(\partial G^{2}\right) \theta^{1} \theta^{2} \partial_{1}+D^{1} \theta^{1} \theta^{2} \partial_{1}+D^{2} \theta^{1} \theta^{2} \partial_{2}
\end{aligned}
$$

and a basis of generators is given by

$$
\begin{aligned}
& L_{n}=-t^{n+1} \partial_{t}-\frac{n+1}{2} t^{n}\left(\theta^{1} \partial_{1}+\theta^{2} \partial_{2}\right), \\
& T_{n}=t^{n} \theta^{2} \partial_{1} \text { and } W_{n}=t^{n} \theta^{2} \partial_{2}, \\
& B_{n}=t^{n} \theta^{1} \theta^{2} \partial_{t}, \\
& G_{n}^{1}=t^{n+1 / 2}\left(\theta^{1} \partial_{t}-\partial_{1}\right)+\left(n+\frac{1}{2}\right) t^{n-1 / 2} \theta^{1} \theta^{2} \partial_{2}, \\
& G_{n}^{2}=t^{n+1 / 2} \theta^{2} \partial_{t}-\left(n+\frac{1}{2}\right) t^{n-1 / 2} \theta^{1} \theta^{2} \partial_{1}, \\
& D_{n}^{1}=t^{n-1 / 2} \theta^{1} \theta^{2} \partial_{1} \quad \text { and } D_{n}^{2}=t^{n-1 / 2} \theta^{1} \theta^{2} \partial_{2}
\end{aligned}
$$

The conformal weights are given by $d_{\phi}=\frac{1}{2}$ for $D_{m}^{1}$ and $D_{m}^{2}, d_{\phi}=1$ for $T_{m}, W_{m}, B_{m}$, $d_{\phi}=\frac{3}{2}$ for $G_{m}^{1}$ and $G_{m}^{2}$, and of course $d_{\phi}=2$ for $L_{m}$.

The non-trivial commutation relations between the generators are

$$
\begin{array}{ll}
{\left[W_{n}, T_{m}\right]=T_{n+m},} & {\left[W_{n}, D_{m}^{1}\right]=D_{n+m}^{1},} \\
{\left[W_{n}, G_{m}^{1}\right]=-n D_{n+m}^{2},} & {\left[W_{n}, G_{m}^{2}\right]=G_{n+m}^{2}+n D_{n+m}^{1},} \\
{\left[W_{n}, B_{m}\right]=B_{n+m},} & {\left[B_{n}, G_{m}^{1}\right]=G_{n+m}^{2}+n D_{n+m}^{1},} \\
{\left[T_{n}, D_{m}^{2}\right]=-D_{n+m}^{1},} & {\left[T_{n}, G_{m}^{1}\right]=G_{n+m}^{2},} \\
\left\{G_{n}^{1}, G_{m}^{1}\right\}=2 L_{n+m}, & \left\{G_{n}^{1}, G_{m}^{2}\right\}=(m-n) T_{n+m}, \\
\left\{G_{n}^{1}, D_{m}^{1}\right\}=-T_{n+m}+B_{n+m}, & \left\{G_{n}^{1}, D_{m}^{2}\right\}=-W_{n+m}, \\
\left\{G_{n}^{2}, D_{m}^{2}\right\}=B_{n+m} . &
\end{array}
$$

Note that the generators $L_{n}, G_{n}^{i}$ and $T_{n}$ form a subalgebra of $\operatorname{Der}_{\mathscr{D}}\left(A_{2}\right)$ which is a contraction of the $N=2$ ASC algebra. If we start from the algebra (80) and define the generators $\hat{L}_{n}=L_{n}, \hat{G}_{n}^{1}=G_{n}^{1}, \hat{G}_{n}^{2}=\varepsilon G_{n}^{2}, \widehat{T}_{n}=\varepsilon T_{n}$, and compute the commutation 
relations between these generators at the limit $\varepsilon \rightarrow 0$, one finds

$$
\begin{aligned}
{\left[\hat{L}_{n}, \hat{T}_{m}\right]=-m \hat{T}_{n+m} \text { and }\left[\hat{L}_{n}, \hat{G}_{m}^{i}\right]=(n / 2-m) \hat{G}_{n+m}^{i}, } \\
{\left[\hat{T}_{n}, \hat{G}_{m}^{1}\right]=\hat{G}_{n+m}^{2} \text { and }\left[\hat{T}_{n}, \hat{G}_{m}^{2}\right]=0, } \\
\left\{\hat{G}_{n}^{1}, \hat{G}_{m}^{1}\right\}=2 L_{n+m} \text { and }\left\{\hat{G}_{n}^{2}, \hat{G}_{m}^{2}\right\}=0, \\
\left\{\hat{G}_{n}^{1}, \hat{G}_{m}^{2}\right\}=(n-m) \hat{T}_{n+m},
\end{aligned}
$$

which has to be compared with Eqs. (A.5).

- super-derivation algebra $\operatorname{Der}_{\mathscr{D}}\left(A_{2}\right)$ with $\mathscr{D}=a_{0}\left(\theta^{1} \partial_{t}+\partial_{1}\right)$

The super-Kač-Moody algebra obtained from this one-cocycle has the following non-trivial commutation relations:

$$
\begin{aligned}
{\left[T_{a}^{m}, T_{b}^{n}\right] } & =f_{a b}^{c} T_{c}^{m+n}, & {\left[T_{a}^{m},\left(\Theta^{1}\right)_{b}^{n}\right] } & =f_{a b}^{c}\left(\Theta^{1}\right)_{c}^{m+n}, \\
{\left[T_{a}^{m},\left(\Theta^{12}\right)_{b}^{n}\right] } & =f_{a b}^{c}\left(\Theta^{12}\right)_{c}^{m+n}, & {\left[T_{a}^{m},\left(\Theta^{2}\right)_{b}^{n}\right.} & =f_{a b}^{c}\left(\Theta^{2}\right)_{c}^{m+n}+m a_{0} \delta_{m+n, 0} \delta_{a b}, \\
{\left[\left(\Theta^{12}\right)_{a}^{m},\left(\Theta^{1}\right)_{b}^{n}\right] } & =a_{0} \delta_{m+n, 0} \delta_{a b}, & \left\{\left(\Theta^{1}\right)_{a}^{m},\left(\Theta^{2}\right)_{b}^{n}\right\} & =f_{a b}^{c}\left(\Theta^{12}\right)_{c}^{m+n} .
\end{aligned}
$$

The super-derivations compatible with $\mathscr{D}$ are

$$
\begin{aligned}
\mathscr{L}_{\text {even }} & =A \partial_{t}+\frac{1}{2}(\partial A) \theta^{1} \partial_{1}-T \theta^{2}\left(\theta^{1} \partial_{t}-\partial_{1}\right), \\
\mathscr{L}_{\text {odd }} & =G^{1}\left(\theta^{1} \partial_{t}-\partial_{1}\right)+G^{2} \theta^{2} \partial_{t}-\frac{1}{2}\left(\partial G^{2}\right) \theta^{1} \theta^{2} \partial_{1}+k \partial_{2} .
\end{aligned}
$$

A basis of generators is given by

$$
\begin{aligned}
L_{n} & =-t^{n+1} \partial_{t}-\frac{n+1}{2} t^{n} \theta^{1} \partial_{1}, \\
T_{n} & =-t^{n+1 / 2} \theta^{2}\left(\theta^{1} \partial_{t}-\partial_{1}\right), \\
G_{n}^{1} & =t^{n+1 / 2}\left(\theta^{1} \partial_{t}-\partial_{1}\right) \text { and } G_{n}^{2}=t^{n+1} \theta^{2} \partial_{t}-\frac{n+1}{2} t^{n} \theta^{1} \theta^{2} \partial_{1}, \\
c & =\partial_{2} .
\end{aligned}
$$

The conformal weights are $d_{\phi}=\frac{3}{2}$ for $G_{m}^{1}$ and $T_{m}, d_{\phi}=2$ for $G_{m}^{2}$.

The non-trivial communication relations are given by

$$
\begin{aligned}
{\left[T_{n}, G_{m}^{1}\right] } & =2 G_{n+m}^{2}, \\
\left\{G_{n}^{1}, G_{m}^{1}\right\} & =2 L_{n+m}, \quad\left\{G_{n}^{1}, G_{m}^{2}\right\}=\left(\frac{m}{2}-n\right) T_{n+m}, \\
{\left[T_{n}, c\right] } & =-G_{n}^{1}, \quad\left\{G_{n}^{2}, c\right\}=-L_{n} .
\end{aligned}
$$

Notice that the generators $T_{n}$ and $G_{n}^{2}$ do not obey to a "conformal spin-static theorem," i.e. $T_{n}$ (respectively $G_{n}^{2}$ ) is even (bosonic) with a half-integer conformal spin (respectively odd (fermionic) with an integer conformal spin). In this respect, they behave like conformal ghost fields, and the $c$ generator plays the role of the BRST charge.

- super-derivation algebra $\operatorname{Der}_{\mathscr{D}}\left(A_{2}\right)$ with $\mathscr{D}=a_{2} \theta^{2}\left(\theta^{1} \partial_{t}+\partial_{1}\right)+a_{0}\left(\theta^{1} \partial_{t}+\partial_{1}\right)$

The super-Kač-Moody algebra obtained from this one-cocycle has the following non-trivial commutation relations: 


$$
\begin{aligned}
& {\left[T_{a}^{m}, T_{b}^{n}\right]=f_{a b}^{c} T_{c}^{m+n}-a_{2} m \delta_{m+n, 0} \delta_{a b},} \\
& {\left[T_{a}^{m},\left(\Theta^{12}\right)_{b}^{n}\right]=f_{a b}^{c}\left(\Theta^{12}\right)_{c}^{m+n},} \\
& {\left[T_{a}^{m},\left(\Theta^{1}\right)_{b}^{n}\right]=f_{a b}^{c}\left(\Theta^{1}\right)_{c}^{m+n},} \\
& {\left[T_{a}^{m},\left(\Theta^{2}\right)_{b}^{n}\right]=f_{a b}^{c}\left(\Theta^{2}\right)_{c}^{m+n}+m a_{0} \delta_{m+n, 0} \delta_{a b} .}
\end{aligned}
$$

The super-derivations are

$$
\begin{aligned}
\mathscr{L}_{\text {even }} & =A \partial_{t}+\frac{1}{2}(\partial A) \theta^{1} \partial_{1}-T \theta^{2}\left(\theta^{1} \partial_{t}-\partial_{1}\right), \\
\mathscr{L}_{\text {odd }} & =G^{1}\left(\theta^{1} \partial_{t}-\partial_{1}\right)+G^{2} \theta^{2} \partial_{t}-\frac{1}{2}\left(\partial G^{2}\right) \theta^{1} \theta^{2} \partial_{1} .
\end{aligned}
$$

This algebra is a subalgebra of the previous one, where the discrete generator $c$ is set to zero.

- super-derivation algebra $\operatorname{Der}_{\mathscr{D}}\left(A_{3}\right)$ with $\mathscr{D}=a_{3} \partial_{3}$

The super-Kač-Moody algebra obtained from this one-cocycle has the following non-trivial commutation relations:

$$
\begin{aligned}
& {\left[T_{a}^{m}, T_{b}^{n}\right]=f_{a b}^{c} T_{c}^{m+n}, \quad\left[\left(\Theta^{12}\right)_{a}^{m},\left(\Theta^{3}\right)_{b}^{n}\right]=f_{a b}^{c}\left(\Theta^{123}\right)_{c}^{m+n},} \\
& {\left[T_{a}^{m},\left(\Theta^{i}\right)_{b}^{n}\right]=f_{a b}^{c}\left(\Theta^{i}\right)_{c}^{m+n}, \quad\left[\left(\Theta^{23}\right)_{a}^{m},\left(\Theta^{1}\right)_{b}^{n}\right]=f_{a b}^{c}\left(\Theta^{123}\right)_{c}^{m+n},} \\
& {\left[T_{a}^{m},\left(\Theta^{i j}\right)_{b}^{n}\right]=f_{a b}^{c}\left(\Theta^{i j}\right)_{c}^{m+n}, \quad\left[\left(\Theta^{31}\right)_{a}^{m},\left(\Theta^{2}\right)_{b}^{n}\right]=f_{a b}^{c}\left(\Theta^{123}\right)_{c}^{m+n},} \\
& {\left[T_{a}^{m},\left(\Theta^{123}\right)_{b}^{n}\right]=f_{a b}^{c}\left(\Theta^{123}\right)_{c}^{m+n}, \quad\left[\left(\Theta^{31}\right)_{a}^{m},\left(\Theta^{23}\right)_{b}^{n}\right]=-a_{3} \delta_{m+n, 0} \delta_{a b},} \\
& \left\{\left(\Theta^{i}\right)_{a}^{m},\left(\Theta^{j}\right)_{b}^{n}\right\}=f_{a b}^{c}\left(\Theta^{i j}\right)_{c}^{m+n}, \quad\left\{\left(\Theta^{1}\right)_{a}^{m},\left(\Theta^{123}\right)_{b}^{n}\right\}=-a_{3} \delta_{m+n, 0} \delta_{a b} \text {. }
\end{aligned}
$$

The super-derivations are

$$
\begin{aligned}
\mathscr{L}_{\text {even }}= & A \partial_{t}+\frac{1}{2}(\partial A)\left(\theta^{1} \partial_{1}+\theta^{2} \partial_{2}\right)+B\left(\theta^{3} \partial_{3}-\theta^{1} \partial_{1}-\theta^{2} \partial_{2}\right) \\
& +H^{1} \theta^{1} \partial_{3}+H^{2} \theta^{2} \partial_{3}+U\left(\theta^{1} \partial_{1}-\theta^{2} \partial_{2}\right)+T\left(\theta^{1} \partial_{2}-\theta^{2} \partial_{1}\right) \\
& +S\left(\theta^{1} \partial_{2}+\theta^{2} \partial_{1}\right)+K \theta^{1} \theta^{2} \partial_{t}+\frac{1}{2} \partial K \theta^{1} \theta^{2} \theta^{3} \partial_{3}, \\
\mathscr{L}_{\text {odd }}= & G^{1}\left(\theta^{1} \partial_{t}-\partial_{1}\right)+\left(\partial G^{1}\right) \theta^{1} \theta^{2} \partial_{2}+G^{2}\left(\theta^{2} \partial_{t}-\partial_{2}\right)-\left(\partial G^{2}\right) \theta^{1} \theta^{2} \partial_{1} \\
& +W^{1}\left(\theta^{2} \theta^{3} \partial_{3}+2 \theta^{1} \theta^{2} \partial_{1}\right)+W^{2}\left(\theta^{1} \theta^{3} \partial_{3}-2 \theta^{1} \theta^{2} \partial_{2}\right)+D \theta^{1} \theta^{2} \partial_{3} \\
& +E^{1} \partial_{1}+E^{2} \partial_{2}+E^{3} \partial_{3} .
\end{aligned}
$$

$A$ basis of generators is given by

$$
\begin{aligned}
L_{n} & =-t^{n+1} \partial_{t}-\frac{n+1}{2} t^{n}\left(\theta^{1} \partial_{1}+\theta^{2} \partial_{2}\right), \\
B_{n} & =t^{n}\left(\theta^{3} \partial_{3}-\theta^{1} \partial_{1}-\theta^{2} \partial_{2}\right), \\
H_{n}^{1} & =t^{n-1 / 2} \theta^{1} \partial_{3} \text { and } H_{n}^{2}=t^{n-1 / 2} \theta^{2} \partial_{3}, \\
U_{n} & =t^{n}\left(\theta^{1} \partial_{1}-\theta^{2} \partial_{2}\right), T_{n}=t^{n}\left(\theta^{1} \partial_{2}-\theta^{2} \partial_{1}\right), \quad S_{n}=t^{n}\left(\theta^{1} \partial_{2}+\theta^{2} \partial_{1}\right), \\
K_{n} & =t^{n} \theta^{1} \theta^{2} \partial_{t}+\frac{n}{2} t^{n-1} \theta^{1} \theta^{2} \theta^{3} \partial_{3}, \\
G_{n}^{1} & =t^{n+1 / 2}\left(\theta^{1} \partial_{t}-\partial_{1}\right)+\left(n+\frac{1}{2}\right) t^{n-1 / 2} \theta^{1} \theta^{2} \partial_{2}, \\
G_{n}^{2} & =t^{n+1 / 2}\left(\theta^{2} \partial_{t}-\partial_{2}\right)-\left(n+\frac{1}{2}\right) t^{n-1 / 2} \theta^{1} \theta^{2} \partial_{1}, \\
W_{n}^{1} & =t^{n-1 / 2}\left(\theta^{2} \theta^{3} \partial_{3}+2 \theta^{1} \theta^{2} \partial_{1}\right) \quad \text { and } \quad W_{n}^{2}=t^{n-1 / 2}\left(\theta^{1} \theta^{3} \partial_{3}-2 \theta^{1} \theta^{2} \partial_{2}\right), \\
D_{n} & =t^{n-1} \theta^{1} \theta^{2} \partial_{3}, \\
E_{n}^{1} & =t^{n+1 / 2} \partial_{1}, \quad E_{n}^{2}=t^{n+1 / 2} \partial_{2}, \quad E_{n}^{3}=t^{n} \partial_{3} .
\end{aligned}
$$


The conformal weights are $d_{\phi}=0$ for $D_{m}, d_{\phi}=\frac{1}{2}$ for $W_{m}^{1}, W_{m}^{2}, H_{m}^{1}$ and $H_{m}^{2}, d_{\phi}=1$ for $T_{m}, U_{m}, S_{m}, B_{m}, K_{m}, E^{3}, d_{\phi}=\frac{3}{2}$ for $G_{m}^{1}, G_{m}^{2}, E_{m}^{1}, E_{m}^{2}$.

The non-trivial commutation relations between the generators are

$$
\begin{aligned}
& {\left[T_{n}, S_{m}\right]=2 U_{n+m},} \\
& {\left[U_{n}, S_{m}\right]=2 T_{n+m},} \\
& {\left[T_{n}, U_{m}\right]=-2 S_{n+m},} \\
& {\left[T_{n}, H_{m}^{1}\right]=-H_{n+m}^{2},} \\
& {\left[B_{n}, K_{m}\right]=-2 K_{n+m} \text {, }} \\
& {\left[S_{n}, H_{m}^{1}\right]=H_{n+m}^{2} \text {, }} \\
& {\left[T_{n}, H_{m}^{2}\right]=H_{n+m}^{1} \text {, }} \\
& {\left[S_{n}, H_{m}^{2}\right]=H_{n+m}^{1} \text {, }} \\
& {\left[U_{n}, H_{m}^{1}\right]=H_{n+m}^{1},} \\
& {\left[U_{n}, H_{m}^{2}\right]=-H_{n+m}^{2} \text {, }} \\
& {\left[B_{n}, H_{m}^{1}\right]=-2 H_{n+m}^{1} \text {, }} \\
& {\left[B_{n}, H_{m}^{2}\right]=-2 H_{n+m}^{2} \text {, }} \\
& {\left[T_{n}, G_{m}^{1}\right]=G_{n+m}^{2} \text {, }} \\
& {\left[T_{n}, W_{m}^{1}\right]=W_{n+m}^{2} \text {, }} \\
& {\left[T_{n}, G_{m}^{2}\right]=-G_{n+m}^{1},} \\
& {\left[T_{n}, W_{m}^{2}\right]=-W_{n+m}^{1} \text {, }} \\
& {\left[T_{n}, E_{m}^{1}\right]=-E_{n+m}^{2} \text {, }} \\
& {\left[S_{n}, G_{m}^{1}\right]=G_{n+m}^{2} \text {, }} \\
& {\left[S_{n}, W_{m}^{1}\right]=W_{n+m}^{2} \text {, }} \\
& {\left[T_{n}, E_{m}^{2}\right]=E_{n+m}^{1} \text {, }} \\
& {\left[S_{n}, G_{m}^{2}\right]=G_{n+m}^{1},} \\
& {\left[S_{n}, W_{m}^{2}\right]=W_{n+m}^{1},} \\
& {\left[S_{n}, E_{m}^{1}\right]=-E_{n+m}^{2} \text {, }} \\
& {\left[U_{n}, G_{m}^{1}\right]=G_{n+m}^{1}+2 E_{n+m}^{1},} \\
& {\left[S_{n}, E_{m}^{2}\right]=-E_{n+m}^{1},} \\
& {\left[U_{n}, G_{m}^{2}\right]=-G_{n+m}^{2}-2 E_{n+m}^{2} \text {, }} \\
& {\left[U_{n}, W_{m}^{1}\right]=-W_{n+m}^{1},} \\
& {\left[U_{n}, W_{m}^{2}\right]=W_{n+m}^{2},} \\
& {\left[U_{n}, E_{m}^{1}\right]=-E_{n+m}^{1},} \\
& {\left[U_{n}, E_{m}^{2}\right]=E_{n+m}^{2},} \\
& {\left[B_{n}, G_{m}^{1}\right]=-G_{n+m}^{1}-n W_{n+m}^{2},} \\
& {\left[B_{n}, W_{m}^{1}\right]=-W_{n+m}^{1} \text {, }} \\
& {\left[B_{n}, G_{m}^{2}\right]=-G_{n+m}^{2}-n W_{n+m}^{1},} \\
& {\left[B_{n}, E_{m}^{1}\right]=E_{n+m}^{1} \text {, }} \\
& {\left[B_{n}, E_{m}^{3}\right]=-E_{n+m}^{3} \text {, }} \\
& {\left[B_{n}, W_{m}^{2}\right]=-W_{n+m}^{2} \text {, }} \\
& {\left[B_{n}, E_{m}^{2}\right]=E_{n+m}^{2} \text {, }} \\
& {\left[B_{n}, D_{m}\right]=-3 D_{n+m},} \\
& {\left[K_{n}, G_{m}^{1}\right]=-\left[K_{n}, E_{m}^{1}\right]=G_{n+m}^{2}+E_{n+m}^{2}+\frac{n}{2} W_{n+m}^{1},} \\
& {\left[K_{n}, G_{m}^{2}\right]=-\left[K_{n}, E_{m}^{2}\right]=-G_{n+m}^{1}-E_{n+m}^{1}-\frac{n}{2} W_{n+m}^{2},} \\
& {\left[H_{n}^{i}, G_{m}^{j}\right]=\varepsilon_{i j}(n+m) D_{n+m}+\delta_{i j} E_{n+m}^{3}, \quad\left[H_{n}^{2}, W_{m}^{2}\right]=3 D_{n+m},} \\
& {\left[H_{n}^{1}, W_{m}^{1}\right]=-3 D_{n+m},} \\
& {\left[H_{n}^{1}, E_{m}^{1}\right]=\left[H_{n}^{2}, E_{m}^{2}\right]=-E_{n+m}^{3}} \\
& \left\{G_{n}^{1}, G_{m}^{1}\right\}=\left\{G_{n}^{2}, G_{m}^{2}\right\}=2 L_{n+m}, \quad\left\{G_{n}^{1}, G_{m}^{2}\right\}=(n-m) T_{n+m}, \\
& \left\{G_{n}^{1}, E_{m}^{1}\right\}=-L_{n+m}-\frac{n-m}{2} U_{n+m}, \quad\left\{G_{n}^{2}, E_{m}^{1}\right\}=\frac{n+m}{2} T_{n+m}-\frac{n-m}{2} S_{n+m}, \\
& \left\{G_{n}^{1}, E_{m}^{2}\right\}=-\frac{n+m}{2} T_{n+m}-\frac{n-m}{2} S_{n+m}, \quad\left\{G_{n}^{2}, E_{m}^{2}\right\}=-L_{n+m}+\frac{n-m}{2} U_{n+m}, \\
& \left\{G_{n}^{1}, E_{m}^{3}\right\}=m H_{n+m}^{1}, \quad\left\{G_{n}^{2}, E_{m}^{3}\right\}=m H_{n+m}^{2}, \\
& \left\{G_{n}^{1}, D_{m}\right\}=H_{n+m}^{2}, \quad\left\{G_{n}^{2}, D_{m}\right\}=-H_{n+m}^{1},
\end{aligned}
$$




$$
\begin{aligned}
& \left\{G_{n}^{1}, W_{m}^{1}\right\}=T_{n+m}-S_{n+m}+2 K_{n+m} \\
& \left\{G_{n}^{1}, W_{m}^{2}\right\}=-U_{n+m}-B_{n+m} \\
& \left\{E_{n}^{1}, W_{m}^{1}\right\}=-T_{n+m}+S_{n+m} \\
& \left\{E_{n}^{1}, W_{m}^{2}\right\}=-U_{n+m}+B_{n+m} \\
& \left\{E_{n}^{3}, W_{m}^{1}\right\}=-H_{n+m}^{2} \\
& \left\{E_{n}^{1}, D_{m}\right\}=H_{n+m}^{2}
\end{aligned}
$$$$
\begin{aligned}
& \left\{G_{n}^{2}, W_{m}^{1}\right\}=U_{n+m}-B_{n+m} \\
& \left\{G_{n}^{2}, W_{m}^{2}\right\}=-T_{n+m}-S_{n+m}-2 K_{n+m}, \\
& \left\{E_{n}^{2}, W_{m}^{1}\right\}=U_{n+m}+B_{n+m} \\
& \left\{E_{n}^{2}, W_{m}^{2}\right\}=T_{n+m}+S_{n+m}, \\
& \left\{E_{n}^{3}, W_{m}^{2}\right\}=H_{n+m}^{1}, \\
& \left\{E_{n}^{2}, D_{m}\right\}=H_{n+m}^{1} .
\end{aligned}
$$

Acknowledgements. Special thanks are due to R. Stora for numerous fruitful discussions and suggestions and for a careful reading of the manuscript. It is also a pleasure to thank F. Delduc and J.L. Petersen for discussions on the $N=2$ superconformal algebra and the members of the theory group at LAPP for their strong encouragements.

\section{References}

1. Kač, V. G., Todorov, I. T.: Superconformal current algebras and their unitary representations, Commun. Math. Phys. 102, 337 (1985)

2. Ademollo et al.: Phys. Lett. 62B, 105 (1976); Nucl. Phys. B114, 297 (1976); Ademollo et al.: Nucl. Phys. B111, 77 (1976)

3. Kač, V. G.: Infinite dimensional Lie algebras. Cambridge: Cambridge University Press 1985

4. Pressley, A., Segal, G.: Loop groups. Oxford: Clarendon Press Oxford 1986

5. Connes, A.: Noncommutative differential geometry. Publ. Math. IHES, 62 (1985)

6. Loday, J. L., Quillen, D.: Cyclic homology and the Lie algebra of matrices. Comments Math. Helvetica 59 (1984)

7. Kastler, D.: Cyclic cohomology within the differential envelope. Coll. Travaux en cours. no. 30 Hermann, Paris (1988)

8. Coquereaux, R.: Non-commutative geometry and Theoretical Physics. J. Geom. Phys. 6, 425 (1990)

9. Greub, W., Halperin, S., Vanstone, R.: Connections, Curvature and Cohomology. New York: Academic Press 1972

10. Kassel, C.: A Künneth formula for the cyclic cohomology of $Z_{2}$ graded algebras. Math. Ann. 275, 683 (1986)

11. Karoubi, M.: Formule de Künneth en cohomologie cyclique. C.R.A.S. Serie I, Math 303 no. 12, p. 527 (1986); ibid 13, 595 (1986)

12. Cartan, H., Eilenberg, S.: Homological Algebra. Chap. IX, Princeton, NJ: Princeton University Press

13. Coquereaux, R., Jadczyk, A., Kastler, D.: Differential and Integral Calculus on Grassmann algebras: A review. CPT-2223 (1989)

14. Sevrin, A., Spindel, Ph., Troost, W., Van Proeyen, A.: Nucl. Phys. B308, 465 (1988); Sevrin, A., Troost, W., Van Proeyen, A.: Phys. Lett. B208, 447 (1988)

15. Hasiewicz, Z., Thielemans, K., Troost, W.: J. Math. Phys. 31, 744 (1990)

16. Witten, E.: Non-abelian bosonization in two dimensions. Commun. Math. Phys. 92, 455 (1984); Knizhnik, V. G., Zamolodchikov, A. B.: Current algebra and Wess-Zumino model in two dimensions. Nucl. Phys. B247, 83 (1984)

17. DiVecchia, P., Knizhnik, V. G., Petersen, J. L., Rossi, P.: A supersymmetric Wess-Zumino Lagrangian in two dimensions. Nucl. Phys. B253, 701 (1985) 
